\title{
Minimally complex exchange mechanisms: Emergence of prices, markets, and money $(\mathrm{R})^{*}$
}

\author{
Pradeep Dubey ${ }^{\dagger}$ Siddhartha Sahi $;$ and Martin Shubik ${ }^{\S}$
}

20 April 2014

\section{Abstract}

We consider abstract exchange mechanisms wherein individuals submit "diversified" offers in $m$ commodities, which are then redistributed to them. Our first result is that if the mechanism satisfies certain natural conditions embodying "fairness" and "convenience" then it admits unique prices, in the sense of consistent exchange-rates across commodity pairs $i j$ that equalize the valuation of offers and returns for each individual.

We next define integers $\tau_{i j}, \pi_{i j}$ and $k_{i}$ which represent the "time" required to exchange $i$ for $j$, the "difficulty" in determining the exchange ratio, and the "dimension" of the offer space in $i$; and refer to these as time-, priceand message-complexity of the mechanism. Our second result is that there are only a finite number of minimally complex mechanisms, which moreover correspond to certain directed graphs $G$ in a precise sense. The edges of $G$ can be regarded as markets for commodity pairs, and prices play a stronger role in that the return to a trader depends only on his own offer and the prices.

*In honor of Lloyd Shapley. (This is a slightly revised version of an earlier Stony Brook Working Paper, as well as Cowles Foundation Discussion Paper 1945, both dated 8 April 2014.)

$\dagger$ Center for Game Theory, Department of Economics, Stony Brook University; and Cowles Foundation for Research in Economics, Yale University

${ }^{\ddagger}$ Department of Mathematics, Rutgers University, New Brunswick, New Jersey

$\S$ Cowles Foundation for Research in Economics, Yale University; and Santa Fe Institute, New Mexico. 
Finally we consider "strongly" minimal mechanisms, with smallest "worst case" complexities $\tau=\max \tau_{i j}$ and $\pi=\max \pi_{i j}$. Our third main result is that, for $m>3$ commodities, there are precisely three such mechanisms, which correspond to the star, cycle, and complete graphs, and have complexities $(\pi, \tau)=(4,2),(2, m-1),\left(m^{2}-m, 1\right)$ respectively. Unlike the other two mechanisms, the star mechanism has a distinguished commodity - the money - that serves as the sole medium of exchange. As $m \rightarrow \infty$ it is the only mechanism with bounded $(\pi, \tau)$.

JEL Classification: C70, C72, C79, D44, D63, D82.

Keywords: exchange mechanism, minimal complexity, prices, markets, money.

\section{Introduction}

The early history of human settlement is intimately connected with economic specialization and the concomitant need to exchange commodities, which led to the formation of urban communities where such trades might be better effected. These communities were in regular conflict with one another, with the successful ones progressing from villages to cities to states. Many ancient civilizations seemed to have followed a similar evolutionary path and to have further developed the notion of "money" - a commodity that served as a medium of exchange. The Sumerians used barley, silver, and gold at various stages. Other societies have used cowrie shells, beads, or even large stone disks. In modern times money mostly takes the form of paper, deemed sufficient to settle private and public debt by fiat of the government.

The central question inevitably arises: what are the imperatives that lead to the emergence of a money in an exchange economy? This has been explored in the literature in terms of overcoming frictions in trade, such as the difficulty of "a double coincidence of wants" ${ }^{1}$ or transactions costs (see section 1.3 for a survey). Much of the analyses have been in the framework of a group of sophisticated individuals who maximize utilities in competitive interaction with one another in an economic equilibrium.

Our aim is to show that there is a more elementary rationale for the emergence of money, based on considerations that arise prior to the onset of utilitarianism. To the extent that different urban communities may have

\footnotetext{
${ }^{1}$ In pairwise encounters between individuals, a trader must have the rare good fortune to meet another who not only has what he wants, but also wants what he has.
} 
developed different mechanisms of exchanging goods, one can fruitfully apply the idea of competition to the "exchange mechanisms" themselves. Thus one may ask, what attributes of a mechanism might provide it a competitive advantage over others? In this paper we focus on two such attributes, namely "fairness" and "convenience" or "ease of use".

We start with a mechanism stripped down to its bare minimum, leaving only what is necessary to enable trade in a fixed finite set $\{1, \ldots, m\}$ of commodities. The mechanism takes in offers, possibly diversified, of each commodity from an arbitrary set of individuals and then redistributes back to them everything that it has received. We impose five conditions on the mechanism that we term anonymity, aggregation, invariance, non-dissipation and flexibility, which reflect the twin attributes of fairness and convenience. Although there are infinitely many mechanisms satisfying these conditions, our first result is that every such mechanism admits unique prices, in the sense of consistent exchange-rates across commodity pairs that equalize the valuation of offers and returns for each individual.

We next define some natural notions of "complexity" for a mechanism and, in keeping with the idea of convenience, we study mechanisms with minimal complexity. Our second result is that there are only a finite number of minimal mechanisms, and these moreover have a very special graphical structure. Markets emerge for various commodities, and prices play a stronger role in that the return to a trader depends only on his own offer and the prices.

Finally we introduce certain refined notions of complexity for this finite class and study the corresponding minimal mechanisms, which we term strongly minimal. It turns out that there are only three strongly minimal mechanisms, up to a relabeling of commodities. In one of these, a single commodity emerges endogenously as money and mediates trade among decentralized markets for the other commodities. Moreover, with a moderate increase in the number of commodities, the money mechanism quickly supersedes the other two in a very precise sense.

Note that our analyis addresses the question: "Why money?" It is totally silent on: "What money?" There is considerable discussion in the classical literature regarding the different criteria for the choice of a suitable "commodity money" such as its portability, verifiability, divisibility and durability; or, alternatively, the backing of the state requisite to sustain "fiat money" (see, e,g., [17], [19], [23], [24], [27], [37]; and, for a recent survey on both kinds of money, see [42] and [43]). Our analysis is quite compatible with this 
literature $^{2}$, being only at pains to point out the urgency of appointing some money. In the absence of money, as most tellingly recounted by Jevons [17], matters may get really out of hand!

"Some years since, Mademoiselle Zélie, a singer of the Théâtre Lyrique at Paris, made a professional tour round the world, and gave a concert in the Society Islands. In exchange for an air from Norma and a few other songs, she was to receive a third part of the receipts. When counted, her share was found to consist of three pigs, twenty-three turkeys, forty-four chickens, five thousand cocoa-nuts, besides considerable quantities of bananas, lemons, and oranges. At the Halle in Paris, as the prima donna remarks in her lively letter, printed by M. Wolowski, this amount of live stock and vegetables might have brought four thousand francs, which would have been good remuneration for five songs. In Society Islands, however, pieces of money were very scarce; and as Mademoiselle could not consume any considerable portion of the receipts herself, it became necessary in the mean time to feed the pigs and poultry with the fruit."

The discussion we shall present will be somewhat dry and mathematical, and certainly lacking in the liveliness of Mademoiselle Zélie's recollections, but we hope that it may serve as a useful supplement. For the convenience of the reader we provide an outline of our model and results in somewhat greater detail in the next two subsections, postponing the formal discussion till section 2 .

\subsection{Outline of the Model}

Our analysis is carried out in the spirit ${ }^{3}$ of mechanism-design, with the aim - as was said - of rendering trade as fair and as convenient as possible.

\footnotetext{
${ }^{2}$ Our model can equally accomodate fiat money or commodity money, depending on how preferences are introduced. Indeed, all we suppose is that the $m$ items being traded are distinguishable from one another. In particular, offers could just be quotes (think of e-commerce!), instead of actual shipment of goods; in which case the mechanism is quoting back what each individual is entitled to receive. Our model leaves the choice open as to whether the quoted promises of delivery and the entitlements due are to be netted, or not; and what penalties need be levied for default in deliveries.

${ }^{3}$ However we are not trying to implement, via dominant or Nash strategies, any desired "solution" on a given domain of indviduals' characteristics, as is common in much of the mechanism-design literature. In our framework, there are no such characteristics to begin with; nor therefore any solution emanating from them. We use the word "mechanism" (see section 1.1.3.1 of [26]) "with its "plain english" meaning instead of the meaning it has been given in technical parlance." And its express purpose is to enable everyman to trade,
} 
To enable individuals to play a more influential role in the drama of trade beyond just their offer of commodities, we introduce a common language in which they may communicate with the mechanism $M$. No structure is imposed on the language except that it be of finite size. There is, for each commodity $i$, an abstract finite ${ }^{4}$ set $K_{i}=K_{i}(M)$, whose elements may be thought of as costless messages that accompany offers in $i$. The elements of $K_{i}$ thus serve to diversify the offers in $i$, and that is their sole purpose. To emphasize the abstract nature of the language, we use the agnostic name $i$-index for an element of $K_{i}$. It is our purpose to see how far matters can develop with the use of such a language. The reader may find the following concrete analogy useful to keep our abstract scenario in mind. Imagine a set $K_{i}$ of "bins" made available for each commodity $i$. An "elementary action" consists of depositing a quantity of $i$ into one of the bins in $K_{i}$. Based upon the entire conglomeration of elementary actions, the mechanism $M$ assigns a return vector in $\mathbb{R}_{+}^{m}$ to every such action, and in the process sends back all the commodities it has received, emptying out the bins. An individual is, of course, free to take as many actions as he wants and add up the returns that the mechanism sends back for each of his actions.

Fixing a mechanism $M$, the overall offer of commodities by an individual may be represented, for expositional convenience, by a vector in ${ }^{5} \mathbb{R}_{+}^{K}$, where $K=K_{1} \amalg \cdots \amalg K_{m}$ denotes the disjoint union of the $K_{i}$. (It will shortly become evident, in view of the aggregation condition we impose below on $M$, that this is tantamount to allowing the individual to take any finite number of "elementary actions" that were referred to earlier.) The mechanism, as was said, then redistributes the offers made by all the individuals in the population, sending back to each a return vector in $\mathbb{R}_{+}^{m}$, and conserving commodities in the process. The collection of maps from $n$-offers to $n$-returns (one map for each $n$ ) constitutes $^{6}$ the mechanism $M$.

Our five conditions on mechanisms are as follows.

with the simple expedient of offering commodities and without having to account for his precise motivation or even bothering to pretend that he has one.

${ }^{4}$ As we vary $M$ the cardinality of $K_{1}, \ldots, K_{m}$ ranges over all $m$-tuples of positive integers, thus there is no a priori upper bound on the size of the language.

${ }^{5}$ For any finite set $X$, we denote by $A^{X}$ the set of maps from $X$ to $A$. Thus $\mathbb{R}^{X}$ is the Euclidean space whose axes are indexed by the elements of $X$; and $\mathbb{R}_{+}^{X}$ and $\mathbb{R}_{++}^{X}$ are its non-negative and strictly positive orthants. When $X=\{1, \ldots, m\}$ is the set of commodities, we write $\mathbb{R}^{m}$ etc. for brevity (since commodities are fixed throughout).

${ }^{6}$ When there is a continuum of traders, our analysis goes through mutatis mutandis after making the obvious changes (see section 10). 
The first condition, anonymity, stipulates that the mechanism be blind to all characteristics of a trader other than his offer. In other words, any two traders who send in the same offer are assigned the same returns.

The second condition, aggregation, says that if a trader pretends to be two different persons by splitting his offer, others' returns are unaffected.

If either of these conditions were violated, trade would become a cumbersome affair: each individual would need to keep track of the full distribution of offers across the entire population, and then figure out how to diversify his own offers in response. Thus these conditions contribute to convenience in trade. They also embody fairness, enabling free entry for any new participant on non-discriminatory terms, and thereby rendering the mechanism more "inclusive".

It is an immediate consequence of anonymity and aggregation that the return to any individual is a function only of his own offer $a \in \mathbb{R}_{+}^{K}$ and the aggregate $b \in \mathbb{R}_{++}^{K}$ of all offers ${ }^{7}$; moreover this function $r(a, b)$ is the same for all individuals. (In light of this fact, we shall call the aggregate vector $b$ the state of the mechanism.). We define the net trade function to be $\nu(a, b)=r(a, b)-\bar{a}$, where $\bar{a} \in \mathbb{R}_{+}^{m}$ is the vector of commodities "used up" in making the offer $a \in \mathbb{R}_{+}^{K}$.

The third condition is invariance. Its main content is that the maps which comprise $M$ are invariant under a change of units in which commodities are measured. This makes the mechanism much simpler to operate in: one does not need to keep track of seven pounds or seven kilograms or seven tons, just the numeral 7 will do.

The fourth condition is non-dissipation and says that no trader's return can be less commodity-wise than his offer, i.e., if $\nu(a, b) \neq 0$, then at least one component of $\nu(a, b)$ must be positive. If it were violated, such unfortunate traders would tend to abandon the mechanism.

To state our final condition we consider the perspective of a binary $i j$ trader $^{8}$, who wishes to interact with the mechanism to exchange a single commodity $i$ for some other commodity $j$. Note that if $a$ is an offer of $i$ in an index $h \in K_{i}$, the return $r(a, b)$ will in general be a commodity bundle, whose composition may depend on the state $b$. If $r(a, b)$ consists exclusively

\footnotetext{
${ }^{7}$ Throughout we shall assume that on aggregate all indices are active, i.e., $b$ is a strictly positive vector. (It will suffice, for our purposes, to characterize the behavior of the mechanisms on this restricted interior domain.)

${ }^{8}$ Binary trades will shortly be seen to form an iterative basis for all trade.
} 
of commodity $j$ for all states $b$ and all ${ }^{9} h$-offers $a$, we will say that $h$ is a "pure" $i j$-index or an $i j$-market. In the absence of such markets, an $i j$-trader may be forced to accept commodity $j$ bundled with other commodities.

The fifth condition we impose on the mechanism is flexibility. It requires that there are "enough" markets to enable individuals to "unbundle" their returns. More precisely, we require that if $r(a, b)$ has a positive $j$-component for some market state $b$ and some offer $a$ solely in $i$, then the mechanism has an $i j$-market.

\subsection{The Key Results}

We shall identify mechanisms, which are of "minimal complexity" amongst those that satisfy the five conditions above. Two relevant notions of complexity will be developed from the standpoint of binary traders.

A natural concern of such a trader is: what is the minimum number of time periods $\tau_{i j}(M)$ needed to convert $i$ to $j$ ? (The precise definitions of "conversion" and $\tau_{i j}(M)$ are given in section 3.1.) We say that a mechanism is connected if $\tau_{i j}(M)<\infty$ for all $i \neq j$, and we write $\mathfrak{M}=\mathfrak{M}(m)$ for the class of connected mechanisms satisfying the five conditions.

Let $\mathbb{R}_{++}^{m} / \sim$ be the set of rays ${ }^{10}$ in $\mathbb{R}_{++}^{m}$. A price function for a mechanism is a map $p$ from $\mathbb{R}_{++}^{K}$ to $\mathbb{R}_{++}^{m} / \sim$ satisfying value conservation: $p(b) \cdot \nu(a, b)=$ 0 for every $a \leq b$. In other words, prices $p(b)$ are determined by the state $b$ of the mechanism; and the value - under the prevailing prices — of each individual's offer is equal to that of his returns.

We can now state our first result.

\section{Every mechanism of $\mathfrak{M}$ admits a unique price function.}

On account of value conservation, it is evident that binary trades form an iterative basis of all trade for mechanisms in $\mathfrak{M}$, reinforcing our focus on them. Note that value conservation is perforce true on the aggregate since commodities are neither created nor destroyed by the mechanism, only redistributed. Thus what the result essentially shows is that the mechanism does not assign "profitable" trades to some at the expense of others.

\footnotetext{
${ }^{9} \mathrm{By}$ invariance it is enough to require that for each $b$ there exist some such $h$-offer $a$.

${ }^{10} \mathrm{~A}$ ray $p$ represents a price vector up to overall multiplication by a positive scalar; the ratios $p_{i} / p_{j}$ represent well-defined consistent exchange rates across all pairs $i j$ of commodities.
} 
The other major concern of our binary trader is also clear: how much of commodity $j$ can he get per unit of $i$ ? It follows from our first result that he can calculate this in terms of his own offer of $i$ and the state of the mechanism which determines the exchange rate ${ }^{11} p_{i} / p_{j}$. Define $\pi_{i j}(M)$ to be cardinality of the minimal set of components of the state of the mechanism required to compute the function $p_{i} / p_{j}$; equivalently the minimal set of bins that he (or, the mechanism) needs to look into in order to calculate this rate. (For the precise definition, see section 3.3.) The arrays of integers $\tau_{i j}(M)$ and $\pi_{i j}(M)$, as we vary over all distinct pairs $i j$, represent respectively the time complexity and price complexity ${ }^{12}$ of the mechanism. To these arrays we add, by way of a subsidiary consideration, the sizes $k_{i}(M)$ of $K_{i}(M)$ for $1 \leq i \leq m$ which measure message complexity.

Given $M$ and $M^{\prime}$ in $\mathfrak{M}$ with complexities $\tau_{i j}, \pi_{i j}, k_{i}$ and $\tau_{i j}^{\prime}, \pi_{i j}^{\prime}, k_{i}^{\prime}$ respectively, we write $M \preceq M^{\prime}$ if for all $i, j$

$$
\tau_{i j} \leq \tau_{i j}^{\prime}, \quad \pi_{i j} \leq \pi_{i j}^{\prime}, \quad k_{i} \leq k_{i}^{\prime}
$$

Clearly $\preceq$ is reflexive and transitive, and hence constitutes a quasiorder on $\mathfrak{M}$. Let $\mathfrak{M}_{*}=\mathfrak{M}_{*}(m)$ denote the set of $\preceq$-minimal ${ }^{13}$ elements.

For any directed, connected graph $G$ with vertex set $\{1, \ldots, m\}$, one can define a mechanism $M_{G}$ in $\mathfrak{M}$ such that $K_{i}$ is the set of outgoing edges at vertex $i$ (see [8] and section (4.2)). Such " $G$-mechanisms" have very special structure. All the indices are pure, i.e. each edge of $G$ is a market; furthermore, it turns out that prices mediate trade across the markets of $M_{G}$ (see equation (3) in section (4.2)) in the sense mentioned earlier: the return to a trader depends only on his own offer and the prices. Thus prices play the full-fledged role of a "decoupling device" in any $G$-mechanism.

Denote by $\mathfrak{M}_{g}=\mathfrak{M}_{g}(m)$ the finite set of all $G$-mechanisms in $\mathfrak{M}$. We can state our second result.

$\mathfrak{M}_{*}$ is a subset of $\mathfrak{M}_{g}$; in particular, $\mathfrak{M}_{*}$ is a finite set.

\footnotetext{
${ }^{11}$ If there is a continuum of traders, his own action has no effect on the exchange rate and so he can compute the conversion easily. Otherwise he needs to track how his offer alters the state of the market, and thereby the exchange rate. This complication may be ignored, to a first order of approximation, if there are sufficiently many traders in the population.

${ }^{12}$ One could equally have used the term "informational complexity" or — with more accuracy but less panache - "price-informational complexity."

${ }^{13}$ See Definition 50.
} 
Though the mechanisms in $\mathfrak{M}_{*}$ are finite in number, they could be numerous, and so we introduce a finer complexity distinction. Let $\tau(M)=$ $\max \tau_{i j}(M)$ and $\pi(M)=\max \pi_{i j}(M)$ denote the worst-case time complexity and price complexity, and write $M \preceq_{w} M^{\prime}$ if

$$
\pi(M) \leq \pi\left(M^{\prime}\right) \text { and } \tau(M) \leq \tau\left(M^{\prime}\right) .
$$

If $\tilde{\mathfrak{M}}$ is a subset of $\mathfrak{M}$ one can consider the minimal elements of $\tilde{\mathfrak{M}}$ with respect to the quasiorder $\preceq_{w}$ restricted to $\tilde{\mathfrak{M}}$; these will be referred to as strongly minimal mechanisms of $\tilde{\mathfrak{M}}$.

To state our next result we introduce three special mechanisms based on the following graphs, up to relabeling of commodities: the star graph with edges $i m, m i$ for all $i<m$, the cycle graph with edges $12,23, \ldots, m 1$, and the complete graph with edges $i j$, all $i \neq j$.

If ${ }^{14} m>3$ then the three special mechanisms are precisely the strongly minimal mechanisms of both $\mathfrak{M}_{*}(m)$ and $\mathfrak{M}_{g}(m)$. Their complexities are

\begin{tabular}{|c|c|c|c|}
\hline & Star & Cycle & Complete \\
\hline$\pi(M)$ & 4 & 2 & $m(m-1)$ \\
\hline$\tau(M)$ & 2 & $m-1$ & 1 \\
\hline
\end{tabular}

The star mechanism thus either outright dominates any non-star mechanism component-wise (being strictly better in some component, and no worse in the other); or else, it loses by a slight margin in some component, but wins by a huge margin in the other component (the margin of victory going to infinity with $m$ ). An immediate upshot is that if we take any weighted sum $A \pi(M)+B \tau(M)$ as a proxy for total complexity, where $A$ and $B$ are arbitrary positive constants, then the star mechanism will be the unique minimizer of total complexity in $\mathfrak{M}(m)$ for sufficiently large $m$.

\subsection{Related Literature}

The emergence of money and its role in the exchange of commodities has been a matter of considerable discussion in economics. We present some

\footnotetext{
${ }^{14}$ When $m=3$, we get a fourth mechanism with complexities 4,2 identical to the star mechanism. And when $m=2$, we must change 4 to 2 in the table (the three graphs become identical with complexities 2,2 for each).
} 
references that are only indicative, and far from exhaustive. (For a more comprehensive survey, see [37], [42], [43].)

Jevons [17] emphasized four distinct functions of money, which were subsequently popularized as follows in a couplet by Milnes [28]:

"Money's a matter of functions four,

A Medium, a Measure, a Standard, a Store".

While there may be debate on details, the overall categorization of Jevons has survived, even into modern textbooks on macroeconomics, although many authors (see, e.g., [1], [22]) now tend to subsume one of the four functions (the "standard") under the other three. However, as Jevons himself pointed out, the "medium of exchange" function provides the logical foundation upon which the others stand (Chapter 3 of [17], italics ours):

"Being accustomed to exchange things frequently for sums of money, people learn the value of other articles in terms of money", with the upshot that that money becomes the unit of account, or "measure of value", for all transactions. In the same vein, referring to the units for deferred payments when credit comes into play, Jevons notes that "it will, of course, be desirable to select as the standard of value that which appears likely to continue to exchange for many other commodities." Finally he observes that to have a "store of value" it is requisite that whatever is put into storage should be usable, possibly upon liquidation, as a medium of exchange when it is retrieved; and hence "the current money of a country is perhaps more likely to fulfil these conditions than anything else, although diamonds and other precious stones, and articles of exceptional beauty and rarity, might occasionally be employed".

Several search-theoretic models, involving random bilateral meetings between long-lived agents, have been developed following Jevons [17] (see, e.g., [2], [16], [18], [20], [21], [25], [29], [45] and the references therein). These models turn on utility-maximizing behavior and beliefs of the agents in Nash equilibrium, and shed light on which commodities are likely to get adopted as money. A parallel, equally distinctive, strand of literature builds on partial or general equilibrium models with other kinds of frictions in trade, such as limited trading opportunities in each period, or transactions costs (see, e.g., [11], [12], [13], [14], [15], [30], [31], [43], [44], [46]). In each of these models, a specific trading mechanism is exogenously fixed, and the focus is on activity within the mechanism that is induced by equilibrium, based again upon optimal behavior of utilitarian individuals. 
Our approach complements this literature in two salient ways, and brings to light a new rationale for money that is different from those propounded earlier, but not inimical to them, in that the door is left fully open to incorporate their concerns within our framework. First, as we have emphasized, our focus is purely on mechanisms of trade with no regard to the characteristics of the individuals such as their endowments, production technologies, preferences or beliefs. Second, no specific trading mechanism is specified exante by us. We start with a welter of mechanisms and cut them down by complexity considerations, ultimately ending up with the star mechanism.

The model we present builds squarely upon [8], which provided an axiomatic characterization of the finite set of "G-mechanisms" (see section 4.2), bridging the gap between the Shapley-Shubik model of decentralized "trading posts" (see [38], [39], [40]) and the Shapley model of centralized "windows" (see [36]). Various strategic market games, based upon trading posts (the star mechanism), have been analysed, with commodity or fiat money in [4], [32], [33], [34], [38], [39], [40], [41]; many of these papers also discuss the convergence of Nash equilibria (NE) to Walras equilibria (WE) under replication of traders. For a continuum-of-traders version of these models, with details on explicit properties of the commodity money (its distribution and desirability) or of fiat money (its availability and the harshness of default penalties), which guarantee equivalence (or near-equivalence) of NE and WE, see [7], [9], [10]; and, for an axiomatic approach to the equivalence phenomenon, see $[5]$.

Strategic market games differ in a fundamental sense from the Walras equilibrium model, despite the equivalence of $\mathrm{NE}$ and WE. In the WE framework, agents always optimize generating supply and demand functions, but markets do not clear except at equilibrium. We are left in the dark as to what happens outside of equilibrium. In sharp contrast markets always clear, producing prices and trades based on agents' strategies, in the market games; but agents do not optimize except at equilibrium. The very formulation of a game demands that the "game form", i.e., the map from strategies to outcomes, must be defined prior to the introduction of agents' preferences on outcomes; thus disentangling the physics of trade from its psychology. Our mechanisms are firmly in this genre, and indeed form the bases upon which many market-games are built. To be precise: game forms arise from our mechanisms by introducing private endowments, along with the constraints that these impose on individuals' offers; and market games then arise by further introducing preferences. 
In conclusion, let us reiterate that our purpose here is to deduce the existence of prices, markets and money in the simplest possible mechanism. To this end we start with the minimalistic postulate that quantities of commodities are offered in trade; adding on only a rudimentary syntax whose sole intent is to enable traders to diversify their offers. Once we have prices, more sophisticated strategies can come into being, wherein agents use prices alongside quantities in a semantic sense, in order to make contingent statements and thereby protect themselves against vagaries of the market. For extensions in this direction of the Shapley-Shubik trading-posts game form, see [6]; and for the much more complex extension of the Shapley-windows game form, see [26]. It may well be that a unified abstract approach exists, which encompasses these two models, and more, and does within the Bertrandian setting what we have done in the Cournotian, but that is a topic for future exploration.

\section{The Formal Model}

\subsection{Exchange Mechanisms}

We now present the model and the five conditions in a more formal manner. The treatment is the same as in [8], except that we impose the conditions of non-dissipation and flexibility in lieu of "Price Mediation" (see section 4.2) that was used in [8], obtaining a bigger class of mechanisms here.

A exchange mechanism $M$ allows individuals in $\{1, \ldots, n\}$ to trade by means of quantity offers in each commodity in the set $\{1, \ldots, m\}$. Here $m$ is fixed and $n=2,3, \ldots$ can be arbitrary. As discussed in the introduction, we assume that for each commodity $i$, there is a finite set $K_{i}$ of $i$-indices that can accompany offers in $i$. Thus the offer in $i$ can be an arbitrary vector in $\mathbb{R}_{+}^{K_{i}}$ and we define

$$
K=K_{1} \amalg \cdots \amalg K_{m}, \quad S=\mathbb{R}_{+}^{K}, \quad S_{+}=\mathbb{R}_{++}^{K} ;
$$

$S$ (resp. $S_{+}$) is the space of offers (resp., strictly positive offers). Also define

$$
\bar{a}=\left(\overline{a_{1}}, \ldots, \overline{a_{m}}\right)
$$

where $\overline{a_{i}} \in \mathbb{R}_{+}$is the sum of the components of $a_{i} \in \mathbb{R}_{+}^{K_{i}}$, and denotes the total amount of commodity $i$ involved in sending offer $a_{i}$. Let $S^{n}$ be the $n$-fold 
Cartesian product of $S$ with itself, and (with $\boldsymbol{a}=\left(\boldsymbol{a}^{1}, \ldots, \boldsymbol{a}^{n}\right)$ ) let

$$
S(n)=\left\{\boldsymbol{a} \in S^{n}: \sum_{\alpha=1}^{n} \overline{\boldsymbol{a}}^{\alpha} \in S_{+}\right\}
$$

denote the $n$-tuples of offers that are positive on aggregate. Also let $C=\mathbb{R}_{+}^{m}$ denote the commodity space; and $C^{n}$ its $n$-fold product.

An exchange mechanism $M$, on a given set of $m$ commodities, is a collection of maps (one for each positive integer $n$ ) from $S(n)$ to $C^{n}$ such that, if $\boldsymbol{a} \in S(n)$ leads to returns $\mathbf{r} \in C^{n}$, then we have (reflecting the conservation of commodities) :

$$
\sum_{\alpha=1}^{n} \overline{\boldsymbol{a}}^{\alpha}=\sum_{\alpha=1}^{n} \boldsymbol{r}^{\alpha}
$$

\subsubsection{Conditions on the Mechanisms}

In this section give a precise statement of each of the five conditions on a mechanism $M$ that were alluded to in section 1.1.

The first condition is that the mechanism must be blind to all other characteristics of a trader except for his offer:

Condition 1 (Anonymity) Suppose $\boldsymbol{a} \in S(n)$ and $\boldsymbol{a}^{\alpha}=\boldsymbol{a}^{\beta}$. Let $\mathbf{r}$ denote the returns that accrue from $\boldsymbol{a}$. Then $\mathbf{r}^{\alpha}=\mathbf{r}^{\beta}$.

The second condition is that if any trader pretends to be two different persons by splitting his offer, the returns to the others is unaffected. It is easier (and sufficient !) to state this for the "last" trader.

Condition 2 (Aggregation) Suppose $\boldsymbol{a} \in S(n)$ and $\boldsymbol{b} \in S(n+1)$ are such that $\boldsymbol{a}^{\alpha}=\boldsymbol{b}^{\alpha}$ for $\alpha<n$ and $\boldsymbol{a}^{n}=\boldsymbol{b}^{n}+\boldsymbol{b}^{n+1}$. Let $\mathbf{r}, \mathbf{s}$ denote the returns that accrue from $\boldsymbol{a}, \boldsymbol{b}$ respectively. Then $\mathbf{r}^{\alpha}=\boldsymbol{s}^{\alpha}$ for $\alpha<n$.

As remarked before, anonymity and aggregation immediately imply that, regardless of the size $n$ of the population, the return to any trader may be written $r(a, b)$,where $a \in S$ is his own offer and $b \in S_{+}$is the aggregate of all offers. Recall that $\nu(a, b)=r(a, b)-\bar{a}$ denotes his net trade.

For the remaining conditions, it will be useful to introduce some more notation. Let $L \subset P$ and let $w \in \mathbb{R}^{P}$. 
1. We write $\lambda *_{L} w$ for the vector obtained by scaling the $L$-components of $w$ by the scalar $\lambda$

2. We say that a non-zero vector $w$ is an $L$-vector if its non- $L$ components are 0 ; equivalently if $\lambda *_{L} w=\lambda w$

3. By an $\bar{\imath} j$-vector we mean an $\{i, j\}$-vector that has a negative $i$-component, a positive $j$-component.

In what follows, we will apply this notation and speak of $L$-offers and $L$-returns. Also, we will consistently use $a$ for an individual's offer and $b$ for the positive aggregate offer; so, when we refer to the pair $a, b$ it will be implicit that $a \in S, b \in S_{+}$(and also, for the moment, that $a \leq b$; though we shall drop this inequality soon, in view of Proposition 6 below).

Condition 3 (Invariance) $\quad \nu\left(\lambda *_{K_{i}} a, \lambda *_{K_{i}} b\right)=\lambda *_{i} \nu(a, b)$ for all $a, b$ and positive scalars $\lambda$.

The fourth condition is that no trader can get strictly less than his offer.

Condition 4 (Non-dissipation) If $\nu(a, b) \neq 0$, then $\nu_{i}(a, b)>0$ for some component $i$.

Define $h \in K_{i}$ to be an $i j$-index (resp., a pure $i j$-index or an $i j$-market) if there exists an $h$-vector $a \in S$ such that $r_{j}(a, b)>0$ for some $b$ (resp., $r(a, b)$ is a $j$-vector for all $b$.) Our fifth condition is as follows.

Condition 5 (Flexibility) If $M$ has an $i j$-index then it has an ij-market.

As was said, flexibility assures us of the presence of enough $i j$-markets to enable traders to "unbundle" returns.

A mechanism is determined uniquely by its net trade function $\nu(a, b):=$ $r(a, b)-\bar{a}$, which, although initially defined for $a \leq b$ admits a natural extension as follows.

Proposition 6 The net trade $\nu$ admits a unique extension to $S \times S_{+}$satisfying

$$
\nu(\lambda a, b)=\lambda \nu(a, b), \quad \nu(a, \lambda b)=\nu(a, b) \text { for all positive } \lambda
$$

Proof. See Lemma 1 of [8]. Although [8] considers a more restrictive class of mechanisms, we note that the proof of Lemma 1 there only uses anonymity, aggregation, and invariance.

In view of the above result, we drop the restriction $a \leq b$ when considering $\nu(a, b)$. 


\subsubsection{Further Comments on the Conditions}

Aggregation does not imply that if two individuals were to merge, they would be unable to enhance their "oligopolistic power". For despite the aggregation condition, the merged individuals are free to coordinate their actions by jointly picking a point in the Cartesian product of their action spaces. Indeed all the mechanisms we obtain display this "oligopolistic effect", even though they also satisfy aggregation.

It is worthy of note that the cuneiform tablets of ancient Sumeria, which are some of the earliest examples of written language and arithmetic, are in large part devoted to records and receipts pertaining to economic transactions. Invariance postulates the "numericity" property of the maps $r(a, b)$ (equivalently, $\nu(a, b)$ ) making them independent of the underlying choice of units, and this goes to the very heart of the quantitative measurement of commodities. In its absence, one would need to figure out how the maps are altered when units change, as they are prone to do, especially in a dynamic economy. This would make the mechanism cumbersome to use.

Non-dissipation (in conjunction with aggregation, anonymity, and the conservation of commodities) immediately implies no-arbitrage: for any $a, b$ neither $\nu(a, b) \gtreqless 0$ nor $\nu(a, b) \lesseqgtr 0$. To see this, note that in view of Proposition 6 we need consider only the case $a \leq b$ and rule out $\nu(a, b) \supsetneqq 0$. Denote $c=b-a$. Then $\nu(a, b)+\nu(c, b)=\nu(a+c, b)=\nu(b, b)=0$, where the first equality follows from aggregation, and the last from conservation of commodities. But then $\nu(a, b) \nsupseteq 0$ implies $\nu(c, b) \lesseqgtr 0$, contradicting non-dissipation.

Flexibility guarantees the existence of certain $i j$-markets. However the mechanism may well admit complex trading opportunities, such as swaps of commodity bundles, that coexist with the $i j$-markets; the former comprising, so to speak, a tangled web around the latter. It is our complexity criteria below which eliminate the web and allow only the markets to survive, see Theorem 11.

\section{Complexity}

We turn now to the notion of the complexity of such a mechanism. As discussed in the introduction, the idea is to define complexity from the stand- 
point of a "binary" $i j$-trader ${ }^{15}$ who interfaces with $M$ in order to exchange commodity $i$ for commodity $j$. We focus on two basic concerns for such a trader: first, how long will it take him to effect the exchange; and, second, how difficult will it be for him to figure out the terms of exchange? The first concern leads to the notion of "time complexity", and the second to that of "price complexity".

\subsection{Time Complexity}

Definition 7 Given two commodity bundles $v, w \in C$ we will say that $v$ can be converted to $w$, and we write $v \rightarrow w$ if there exist $a, b$ such that

$$
w=v+\nu(a, b) \text { and } \bar{a} \leq v .
$$

We write $\tau(v, w, M)$ for the smallest "time" $t$ for which there is a sequence

$$
v \rightarrow v^{1} \rightarrow \cdots \rightarrow v^{t-1} \rightarrow w .
$$

If $v, w$ are restricted to being $i$ - and $j$ - vectors, then by invariance it follows that the ij-time complexity $\tau_{i j}(M):=\tau(v, w, M)$ is independent of the particular choice of $v, w$. We further define the (maximum) time complexity $\tau(M):=\max _{i \neq j}\left\{\tau_{i j}(M)\right\}$ and say that a mechanism $M$ is connected if $\tau(M)<\infty$.

We denote by $\mathfrak{M}=\mathfrak{M}(m)$ the class of all connected mechanisms with commodity set $\{1, \ldots, m\}$.

\subsection{The Emergence of Prices}

Recall that $\mathbb{R}_{++}^{m} / \sim$ is the set of rays in $\mathbb{R}_{++}^{m}$, representing prices. It turns out that prices emerge in connected mechanisms; and the values, under these prices, of offers and returns are conserved for every trader.

Theorem 8 Let $M$ be connected with associated net trade function $\nu$. Then there is a unique map $p: \mathbb{R}_{++}^{K} \rightarrow \mathbb{R}_{++}^{m} / \sim$ satisfying $p(b) \cdot \nu(a, b)=0$.

\footnotetext{
${ }^{15}$ We focus on bilateral trades between pairs of commodities because they form an iterative basis for all trade. This is so on account of prices (exchange rates) that will shortly be shown to emerge and govern all trade.
} 
Even though $p(b)$ is only defined up to an overall scalar multiple, for each pair $i, j$ we get a well-defined price ratio function

$$
p_{i j}: S_{+} \mapsto \mathbb{R}_{++} ; \quad p_{i j}(b)=\frac{p_{i}(b)}{p_{j}(b)}
$$

Recall the notion of an $\bar{i} j$-vector from section 2.1.1. Theorem 8 has the following immediate consequence.

Corollary 9 Suppose $\nu(a, b)$ is an $\bar{\imath} j$-vector. Then $\frac{\nu_{i}(a, b)}{\nu_{j}(a, b)}=-p_{i j}(b)$.

\subsection{Price Complexity}

Note that a binary $i j$-trader is only interested in net trades $\nu(a, b)$ that are $\bar{\imath} j$-vectors. By the previous corollary, the exchange ratio $\frac{\nu_{i}(a, b)}{\nu_{j}(a, b)}$ is independent of the action $a$ producing the $\bar{\imath} j$-trade, and depends only on $p_{i j}(b)$. Therefore such a trader is interested only in those components of $b$ which "influence" the function $p_{i j}(b)$.

To make this notion precise, say that component $i$ is influential for a function $f\left(x_{1}, \ldots, x_{l}\right)$ if there are two inputs $x, x^{\prime}$, differing only in the $i$ th place, such that $f(x) \neq f\left(x^{\prime}\right)$. Define the $i j$-price complexity $\pi_{i j}(M)$ to be the number of influential components of the function $p_{i j}$. Also define the (maximum) price complexity by

$$
\pi(M):=\max \left\{\pi_{i j}(M): i \neq j\right\}
$$

\section{The Emergence of Markets: G-Mechanisms}

\subsection{Directed Graphs}

In this paper by a graph we mean a directed simple graph. Such a graph $G$ consists of a finite vertex set $V_{G}$, togther with an edge set $E_{G} \subseteq V_{G} \times V_{G}$ that does not contain any loops, i.e., edges of the form $i$. For simplicity we shall often write $i \in G, i j \in G$ in place of $i \in V_{G}, i j \in E_{G}$ but there should be no confusion.

By a path $i i_{1} i_{2} \ldots i_{k} j$ from $i$ to $j$ we mean a nonempty sequence of edges in $G$ of the form

$$
i i_{1}, i_{1} i_{2}, \ldots, i_{k-1} i_{k}, i_{k} j
$$


If $k=0$ then the path consists of the single edge $i j$, otherwise we insist that the intermediate vertices $i_{1}, \ldots, i_{k}$ be distinct from each other and from the endpoints $i, j$. However we do allow $i=j$, in which case the path is called a cycle. We say that $G$ is connected ${ }^{16}$ if for any two vertices $i \neq j$ there is a path from $i$ to $j$.

\subsection{G-mechanisms}

Let $G$ be a connected graph with vertex set $\{1, \ldots, m\}$. Following [8] one may associate to $G$ a mechanism $M_{G} \in \mathfrak{M}(m)$ as follows. We let $K_{i}$ be the set of outgoing edges at vertex $i$, and regard $v \in S$ as a matrix $\left(v_{i j}\right)$ with $v_{i j}$ understood to be 0 if $i j \notin G$. To define $r(a, b)$ we need the following elementary result (see, e.g. [8]).

Lemma 10 For $b \in S_{+}$, there is a unique ray $p=p(b)$ in $\mathbb{R}_{++}^{m} / \sim$ satisfying

$$
\sum_{i} p_{i} b_{i j}=\sum_{i} p_{j} b_{j i} \text { for all } j
$$

Now for $(a, b) \in S \times S_{+}$we set $p=p(b)$ as in (1) and define $r(a, b)$ by

$$
r_{i}(a, b)=p_{i}^{-1}\left(\sum_{j} p_{j} a_{j i}\right) \text { for all } i \text {. }
$$

We remark that the left side of (1) is the total value of all the goods "chasing" good $j$, while the right side is the total value of good $j$ on offer.

Mechanisms of the form $M_{G}$ will be called (connected) $G$-mechanisms, and we write $\mathfrak{M}_{g}=\mathfrak{M}_{g}(m)$ for the totality of such mechanisms. It is worth noting that $\mathfrak{M}_{g}$ is a finite set. Moreover, the formula (2) for the return function of a $G$-mechanism immediately implies

$$
p(b)=p(c) \Longrightarrow r(a, b)=r(a, c) \text { for all } a \in S ; b, c \in S_{+}
$$

In [8] this property was referred to as price mediation and, in conjunction with other axioms, shown to characterize $\mathfrak{M}_{g}$.

\footnotetext{
${ }^{16}$ In [8], the term "irreducible" was used in place of "connected".
} 


\subsection{Minimal Mechanisms}

Given $M$ and $M^{\prime}$ in $\mathfrak{M}$ with complexities $\tau_{i j}, \pi_{i j}, k_{i}$ and $\tau_{i j}^{\prime}, \pi_{i j}^{\prime}, k_{i}^{\prime}$ respectively, we say that $M$ is no more complex than $M^{\prime}$ and write $M \preceq M^{\prime}$ if for all $i, j$

$$
\tau_{i j} \leq \tau_{i j}^{\prime}, \quad \pi_{i j} \leq \pi_{i j}^{\prime}, \quad k_{i} \leq k_{i}^{\prime}
$$

Clearly $\preceq$ is reflexive and transitive, and hence constitutes a quasiorder on $\mathfrak{M}$. We let $\mathfrak{M}_{*}=\mathfrak{M}_{*}(m)$ denote the set of $\preceq$-minimal elements of $\mathfrak{M}$.

Theorem 11 Minimal mechanisms are G-mechanisms: $\mathfrak{M}_{*} \subset \mathfrak{M}_{g}$.

\section{The Emergence of Money}

Let us, from now on, identify two mechanisms if one can be obtained from the other by relabeling commodities. There are three mechanisms of special interest to us in $\mathfrak{M}_{g}(m)$ called the star, cycle, and complete mechanisms; with the following edge-sets:

\begin{tabular}{|c|c|c|c|}
\hline$G$ & Star & Cycle & Complete \\
\hline$E_{G}$ & $\{m i, i m: i<m\}$ & $\{12,23, \ldots, m 1\}$ & $\{i j: i \neq j\}$ \\
\hline
\end{tabular}

Notice that the central vertex $m$ of the graph underlying the star mechanism plays the role of money, and is the sole medium of exchange. ${ }^{17}$

Although the set $\mathfrak{M}_{*}$ is finite, it can be quite large and we will not attempt to characterize it here. Instead we consider the "worst-case complexities" $\pi(M)=\max \pi_{i j}(M)$ and $\tau(M)=\max \tau_{i j}(M)$, and the corresponding quasiorder on $\mathfrak{M}$, namely: $M \preceq_{w} M^{\prime}$ if

$$
\tau(M) \leq \tau\left(M^{\prime}\right), \quad \pi(M) \leq \pi\left(M^{\prime}\right)
$$

If $\tilde{\mathfrak{M}}$ is a subset of $\mathfrak{M}$ one can consider the minimal elements of $\tilde{\mathfrak{M}}$ with respect to the quasiorder $\preceq_{w}$ restricted to $\tilde{\mathfrak{M}}$; these will be referred to as strongly minimal mechanisms of $\tilde{\mathfrak{M}}$.

\footnotetext{
${ }^{17}$ This is reminiscent of "spontaneous symmetry breaking" in physics. The ex ante symmetry between commodities, assumed in our model, is carried over to the cycle and complete mechanisms. It breaks down only in the star mechanism, giving rise to money.
} 
Theorem 12 If $m>3$ then the three special mechanisms are precisely the strongly minimal mechanisms of both $\mathfrak{M}_{*}(m)$ and $\mathfrak{M}_{g}(m)$. Their complexities are

\begin{tabular}{|c|c|c|c|}
\hline & Star & Cycle & Complete \\
\hline$\pi(M)$ & 4 & 2 & $m(m-1)$ \\
\hline$\tau(M)$ & 2 & $m-1$ & 1 \\
\hline
\end{tabular}

The array clearly exhibits the superiority of the star mechanism. As the number of commodities $m$ increases, the other two will beat star slightly in one component, but will lose by a huge margin to star in the other component.

\section{Proof of Theorem 8}

We fix a mechanism $M$ in $\mathfrak{M}$ with net trade function $\nu(a, b)$. Consider the set of pairs $(i, j)$ for which there is at least one $i j$-market (pure $i j$-index) in $K$, and fix a subset $P \subset K$ which contains exactly one $i j$-index for each such pair. Let $S_{P} \subset S$ denote the set of $P$-offers, and define the set of $P$-offers "subordinate" to $v$ as follows:

$$
S_{P}(v)=\left\{a \in S_{P}: \bar{a} \leq v\right\}
$$

Given a vector $v \in S$ we write $\langle v\rangle$ for the class of vectors with the same sign as $v$, thus $w \in\langle v\rangle$ if each component $w_{i}$ has the same sign $(+,-, 0)$ as $v_{i}$.

Lemma 13 Let $v, w \in S$ then the following are equivalent.

1. There is an $a \in S_{P}(v)$ such that $v+\nu(a, b) \in\langle w\rangle$ for some $b \in S_{+}$

2. There is an $a \in S_{P}(v)$ such that $v+\nu(a, b) \in\langle w\rangle$ for all $b \in S_{+}$

3. For each $u \in\langle v\rangle$ there is an $a \in S_{P}(u)$ such that $u+\nu(a, b) \in\langle w\rangle$ for all $b \in S_{+}$

Proof. It is evident that (3) implies (2), and (2) implies (1). We now show that (1) implies (3). Suppose $v, a, b, w$ satisfy (1). Given $u \in\langle v\rangle$ and $b_{*} \in S_{+}$, we need to find $a_{*} \in S_{P}(u)$ such that $u, a_{*}, b_{*}, w$ satisfy (3). Since $u$ and $v$ have the same signs there exist positive scalars $\lambda_{i}$ such that $u_{i}=\lambda_{i} v_{i}$ 
for all $i$. Define $a_{*}$ by $\left(a_{*}\right)_{i}=\lambda_{i} a_{i}$, where (recall) $a_{i}$ is the vector obtained from $a$ by restricting to the $K_{i}$-components. Now we have

$$
\begin{aligned}
v+\nu(a, b) & =(v-\bar{a})+r(a, b) \\
u+\nu\left(a_{*}, b_{*}\right) & =\left(u-\overline{a_{*}}\right)+r\left(a_{*}, b_{*}\right)
\end{aligned}
$$

By construction of $a_{*}$ we have $(v-\bar{a})_{i}=\lambda_{i}\left(u-\overline{a_{*}}\right)_{i}$ for all $i$, and hence $\langle v-\bar{a}\rangle=\left\langle u-\overline{a_{*}}\right\rangle$. Also since $a$ and $a_{*}$ are P-offers, by aggregation and invariance we have $\langle r(a, b)\rangle=\left\langle r\left(a, b_{*}\right)\right\rangle=\left\langle r\left(a_{*}, b_{*}\right)\right\rangle$. We note that if $x, y$ are non-negative vectors then $\langle x+y\rangle$ is uniquely determined by $\langle x\rangle$ and $\langle y\rangle$, thus we get

$$
\left\langle u+\nu\left(a_{*}, b_{*}\right)\right\rangle=\langle v+\nu(a, b)\rangle=\langle w\rangle
$$

which establishes (3).

We note that Lemma $13(3)$ only depends on $\langle v\rangle$ and $\langle w\rangle$ and we will write $\langle v\rangle \rightarrow\langle w\rangle$ if it holds.

Lemma 14 For any $(a, b) \in S \times S_{+}$there is $a_{*} \in S_{P}(\bar{a})$ such that

$$
\langle r(a, b)\rangle=\left\langle\bar{a}+\nu\left(a_{*}, b\right)\right\rangle .
$$

Proof. By aggregation, it suffices to prove this when $a$ is a $K_{i}$-offer for some $i$. By flexibility there is some $a_{*} \in S_{P}(\bar{a})$ such that $r_{i}\left(a_{*}, b\right)=0$, while $r_{j}\left(a_{*}, b\right)$ has the same sign as $r_{j}(a, b)$ for all $j \neq i$. We write

$$
\bar{a}+\nu\left(a_{*}, b\right)=\left(\bar{a}-\overline{a_{*}}\right)+r\left(a_{*}, b\right)
$$

and note that since $a_{*}$ is a pure $K_{i}$-offer, the sign of $r\left(a_{*}, b\right)$ does not change if we rescale $a_{*}$. If $r_{i}(a, b)=0$ we scale up $a_{*}$ to ensure $\overline{a_{*}}=\bar{a}$, while if $r_{j}(a, b)>0$ then we scale down $a_{*}$ to ensure $\overline{a_{*}} \lesseqgtr \bar{a}$; in each case the rescaled $a_{*}$ satisfies (4).

Lemma $15 v^{1} \rightarrow \cdots \rightarrow v^{t}$ implies $\left\langle v^{1}\right\rangle \rightarrow \cdots \rightarrow\left\langle v^{t}\right\rangle$.

Proof. It suffices to show that $v \rightarrow w$ implies $\langle v\rangle \rightarrow\langle w\rangle$. Now by definition

$$
w=v+\nu(a, b) \text { for some }(a, b) \in S \times S_{+} \text {with } \bar{a} \leq v .
$$

If $a_{*}$ is as in (4) then the identities

$$
\begin{aligned}
v+\nu\left(a_{*}, b\right) & =(v-\bar{a})+\left(\bar{a}+\nu\left(a_{*}, b\right)\right) \\
v+\nu(a, b) & =(v-\bar{a})+r(a, b)
\end{aligned}
$$

imply $\left\langle v+\nu\left(a_{*}, b\right)\right\rangle=\langle w\rangle$, whence $\langle v\rangle \rightarrow\langle w\rangle$ by Lemma 13 (1). 
Proposition 16 For $b \in S_{+}$and any $i \neq j$ there is $a \in S_{P}$ such that $\nu(a, b)$ is an $\bar{\imath} j$-vector.

Proof. Let $v$ be an $i$-vector and let $t=\tau_{i j}(M)$ then by definition we have a sequence

$$
v \rightarrow v^{1} \rightarrow \cdots \rightarrow v^{t-1}=w
$$

where $w$ is a $j$-vector. By the previous lemma we get

$$
\langle v\rangle \rightarrow\left\langle v^{1}\right\rangle \rightarrow \cdots \rightarrow\left\langle v^{t-1}\right\rangle \rightarrow\langle w\rangle
$$

By Lemma 13 (3) this means we can find sequences

$$
u^{i} \in\left\langle v^{i}\right\rangle, a^{i} \in S_{P}\left(u^{i}\right) \text { for } i=0, \ldots, t-1
$$

such that $u^{i}+\nu\left(a^{i}, b\right)=u^{i+1}$. If $a=\sum a^{i}$ then we have $a \in S_{P}$ and

$$
\nu(a, b)=\sum \nu\left(a^{i}, b\right)=u^{t}-u^{1}
$$

which is an $\bar{\imath} j$-vector.

It will be convenient to write an $\bar{\imath} j$-vector in the form $(-x, y)$ after suppressing the other components. In the context of the above proposition if $\nu(a, b)=(-x, y)$ then by linearity $\nu(a / x, b)=(-1, y / x)$, and we will say that the offer $a$ (or $a / x$ ) achieves an $i j$-exchange ratio of $y / x$ at $b$.

Lemma 17 If $a^{\prime}, a^{\prime \prime}$ achieve ij-exchange ratios $\alpha^{\prime}, \alpha^{\prime \prime}$ at $b$, then $\alpha^{\prime}=\alpha^{\prime \prime}$.

Proof. By the previous proposition there exists an $a$ such that $\nu(a, b)$ is a $\bar{j} i$-vector; if $\alpha$ is the corresponding exchange ratio then by rescaling $a, a^{\prime}, a^{\prime \prime}$ we may assume that

$$
\nu(a, b)=(1,-\alpha), \nu\left(a^{\prime}, b\right)=\left(-1, \alpha^{\prime}\right), \nu\left(a^{\prime \prime}, b\right)=\left(-1, \alpha^{\prime \prime}\right) .
$$

By Proposition 6 we get

$$
\nu\left(a+a^{\prime}, b\right)=\left(0, \alpha-\alpha^{\prime}\right)
$$

Now by non-dissipation we get $\alpha \geq \alpha^{\prime}$, and exchanging the roles of $i$ and $j$ we conclude that $\alpha^{\prime} \geq \alpha$ and hence that $\alpha=\alpha^{\prime}$. Arguing similarly we get $\alpha=\alpha^{\prime \prime}$ and hence that $\alpha^{\prime}=\alpha^{\prime \prime}$. 
Proof of Theorem 8. Fix $b \in S_{+}$and consider the vector

$$
p=\left(1, p_{2}, \ldots, p_{m}\right)
$$

where $p_{j}^{-1}$ is the $1 j$-exchange ratio at $b$, as in the previous lemma. We will show that $p$ satisfies the conditions of Theorem 8, i.e. that

$$
p \cdot \nu(a, b)=0 \text { for all } a .
$$

We argue by induction on the number $d(a, b)$ of non-zero components of $\nu(a, b)$ in positions $2, \ldots, m$. If $d(a, b)=0$ then $\nu(a, b)=0$ by nondissipation and (5) is obvious. If $d(a, b)=1$ then $\nu(a, b)$ is either an $\overline{1} j$ vector or a $\bar{j} 1$ vector, which by the definition of $p_{j}$ and the previous lemma is necessarily of the form

$$
\left(-x, x p_{j}^{-1}\right) \text { or }\left(x,-x p_{j}^{-1}\right) ;
$$

for such vectors (5) is immediate. Now suppose $d(a, b)=d>1$ and fix $j$ such that $\nu_{j}(a, b) \neq 0$. Then we can choose $a^{\prime}$ such that $\nu\left(a^{\prime}, b\right)$ is a $\overline{1} j$ or a $\bar{j} 1$ - vector such that $\nu_{j}(a, b)=-\nu_{j}\left(a^{\prime}, b\right)$. It follows that $d\left(a+a^{\prime}, b\right)<d$ and by linearity we get

$$
p \cdot \nu(a, b)=p \cdot \nu\left(a+a^{\prime}, b\right)-p \cdot \nu\left(a^{\prime}, b\right) .
$$

By the inductive hypothesis the right side is zero, hence so is the left side.

Finally the uniqueness of the price function is obvious, because the return function of the mechanism dictates how many units of $j$ may be obtained for one unit of $i$, yielding just one possible candidate for the exchange rate for every pair $i j$.

\section{Proof of Theorem 11}

We say a matrix $X$ is an $S \times T$ matrix if its rows and columns are indexed by finite sets $S$ and $T$ respectively; if $Y$ is a $T \times U$ matrix then the product $X Y$ is a well-defined $S \times U$ matrix. For the set $[n]=\{1, \ldots, n\}$ we will speak of $n \times T$ matrices instead of $[n] \times T$ matrices, etc.

Let $M \in \mathfrak{M}(m)$ and write $K_{i}=K_{i}(M)$ and $K=\coprod_{i} K_{i}$ as usual. For any vector $v \in \mathbb{R}_{++}^{m}$, let $D_{v}$ denote the $m \times m$ diagonal matrix $\operatorname{diag}\left\{v_{1}, \ldots, v_{m}\right\}$, and let $E_{v}$ denote the $K \times K$ "extended" diagonal matrix whose $K_{i}$-diagonal entries are all $v_{i}$. Also let $A$ be the $m \times K$ "auxiliary" matrix whose $K_{1^{-}}$ columns are $(1,0, \ldots, 0)^{t}, K_{2}$-columns are $(0,1,0, \ldots, 0)^{t}$, etc. 
Lemma $18 M$ is uniquely determined by a map $b \mapsto N_{b}$ from $S_{+}$to the space of non-negative $m \times K$ column-stochastic matrices as follows.

1. The price ray $p=p(b)$ is obtained as the unique solution of

$$
C_{b} p=\Delta_{b} p
$$

where $\Delta_{b}=A D_{b} A^{t}$ is the diagonal matrix of column sums of $C_{b}=$ $N_{b} D_{b} A^{t}$

2. The return function is given by

$$
r(a, b)=M_{b} a \text { where } M_{b}=D_{p}^{-1} N_{b} E_{p}
$$

Proof. Let $p=p(b)$ be the price function whose existence is guaranteed by Theorem 8 . We will first prove formula 7 for $r(a, b)$ and then prove formula 6. By Proposition 6, the return function of the mechanism $M$ is of the form $r(a, b)=M_{b} a$, where $b \mapsto M_{b}$ is a map from $S_{+}$to the space of non-negative $m \times k$ matrices satisfying

$$
M_{b} b=A b
$$

and the identity

$$
M_{E_{v} b}=D_{v} M_{b} E_{v}^{-1} \text { for all } v \in R_{++}^{m} .
$$

(The non-negativity $M_{b}$ follows from that of $r(a, b)$. The first display holds by conservation of commodities and the second by invariance.) Define

$$
b^{\prime}=E_{p} b, N_{b}=M_{b^{\prime}} .
$$

By invariance it follows that $p\left(b^{\prime}\right)=\mathbf{1}$. Also each column of $N_{b}=M_{b^{\prime}}$ is the return to the offer of a single unit in some commodity. Since all prices are 1 at $b^{\prime}$, Theorem 8 implies that each column of $N_{b}$ sums to 1 , i.e. $N_{b}$ is column stochastic. Now by (8) we get

$$
N_{b}=M_{E_{p} b}=D_{p} M_{b} E_{p}^{-1},
$$

whence $M_{b}=D_{p}^{-1} N_{b} E_{p}$ as desired

Now combining (7) and $M_{b} b=A b$, with the identity $D_{p} A=A E_{p}$ we have

$$
N_{b} E_{p} b=D_{p} M_{b} b=D_{p} A b=A E_{p} b .
$$

Using the identity $E_{p} b=D_{b} A^{t} p$ we can rewrite this as

$$
N_{b} D_{b} A^{t} p=A D_{p} A^{t} b,
$$

which is precisely (6). 
Lemma 19 Let $N_{b}$ in Lemma 18 and let $h \in K_{i}$ be an ij-market.

1. The h-th column of $N_{b}$ is the $j$-th unit vector $e_{j}$, independent of $b$.

2. Every $K_{i}$-column of $N_{b}$ is a linear combination of the "pure" $K_{i}$-columns.

Proof. By definition there is an $h$-offer $a$ such that $r(a, b)=M_{b} a$ is a $j$-return vector. This means that the $h$-th column of $M_{b}$ has a non-zero entry only in its $j$-th component. Since $N_{b}$ is obtained from $M_{b}$ by rescaling entries this is also true of $N_{b}$. By column stochasticity the $h$-th column of $N_{b}$ must be $e_{j}$.

For the second part, let $h^{\prime} \in K_{i}$ be an $i$-index, let $v, w$ be the $h^{\prime}$-th columns of $N_{b}$ and $M_{b}$, and suppose the $j$-th component of $v$ (and hence of $w$ ) is non-zero. It suffices to show that in this case the mechanism has an $i j$-market. However if $a$ is an $h^{\prime}$-offer then $r(a, b)=M_{b} a$ is a multiple of $w$, and thus the assertion follows from flexibility.

Let $G$ be the graph in which we connect $i$ to $j$ if $M$ has an $i j$-market. Since $M$ is connected, Lemma 15 implies that $G$ is connected, and we let $M^{\prime}=M_{G}$ denote the corresponding $G$-mechanism. We will identify the $i$ indices $K_{i}^{\prime}$ of $M^{\prime}$ as a subset of $K_{i}$. If $M$ has several pure $i j$-indices for a given $j$ then this involves a choice, however the choice will play no role in the subsequent discussion. We will refer to $M^{\prime}$ as the embedded $G$-mechanism of $M$.

To continue we need a result from [35]. Let $G$ be any connected directed graph on $\{1, \ldots, n\}$ with weights $z_{i j}$ attached to edges $i j \in G$. We write $Z=\left(z_{i j}\right)$ for the $n \times n$ matrix of edge weights of $G$, setting $z_{i j}=0$ if $i j \notin G$. We also define

$$
\delta_{j}=\sum_{i} z_{i j}, \quad \Delta_{Z}=\operatorname{diag}\left(\delta_{1}, \ldots, \delta_{n}\right),
$$

so that $\Delta$ is the diagonal matrix of column sums of $Z$. We define the weight of a subgraph $\Gamma$ to be the product of its edge weights, thus

$$
w_{\Gamma}(z)=\prod_{i j \in E_{\Gamma}} z_{i j} .
$$

We define an $i$-tree in $G$ to be a (directed) subgraph $T$ with $n$ vertices and $n-1$ edges, and the futher property that $T$ contains a path from $j$ to $i$ for every $j \neq i$. We write $\mathcal{T}_{i}$ for the set of $i$-trees in $G$, and define

$$
w_{i}=\sum_{\Gamma \in \mathcal{T}_{i}} w_{\Gamma}(z), \quad w=\left(w_{1}, \ldots, w_{n}\right)^{t} .
$$


The following lemma from [35] is critical and paves the way for the rest of the analysis.

Lemma 20 If $Z, \Delta_{Z}, w$ are as above then one has $Z w=\Delta_{Z} w$.

We can now prove a key property of embedded $G$-mechanisms.

Proposition 21 If a price ratio depends on some variable in $M^{\prime}$, then it does so in $M$.

Proof. The pure columns of $N_{b}$ are fixed unit vectors, independent of $b$. By assumption there is a bijection between the pure variables and the nonzero entries $c_{r s}(b)$ of the matrix $C_{b}$. We denote the pure components of $b$ by $x=\left(x_{r s}\right)$ and the remaining mixed components by $y=\left(y_{k}\right)$. Then by the definition of $C_{b}$ we have an expression of the form

$$
c_{r s}(b)=x_{r s}+\sum_{k} \varepsilon_{k}(b) y_{k} ; 0 \leq \varepsilon_{k}(b) \leq 1 .
$$

By formula (6) and Lemma 20, the prices $p$ in $M$ and $M^{\prime}$ are weighted sums of trees with edge weights $c_{r s}$ and $x_{r s}$ repectively. Let $p(x, y)$ denote the price vector in $M$ at $b=(x, y)$ and let $p(x)$ denote the price vector in $M^{\prime}$ at $x$. Then by (9) we get

$$
p(x)=\lim _{y \rightarrow 0} p(x, y) .
$$

We now fix a pair of commodities $i, j$ and let $\pi(x, y)$ and $\pi(x)$ denote the price ratios $p_{i} / p_{j}$ in $M$ and $M^{\prime}$ respectively, then we have

$$
\pi(x)=\lim _{y \rightarrow 0} \pi(x, y)
$$

Thus if $\pi(x)$ depends on some $x$-component, so must $\pi(x, y)$.

Proof of Theorem 11. By lemma 15, lemma 19 and the previous proposition (respectively), we have:

$$
\tau_{i j}\left(M^{\prime}\right)=\tau_{i j}(M), k\left(M^{\prime}\right) \leq k(M), \pi_{i j}\left(M^{\prime}\right) \leq \pi_{i j}(M)
$$

If $M$ is minimal then equality must hold throughout. Hence we get $k\left(M^{\prime}\right)=$ $k(M)$ and so $M=M^{\prime}$ is a $G$-mechanism. 


\section{Complexity of $G$-mechanisms}

Let $G$ be a connected graph on $\{1, \ldots, m\}$ as in section 4.2. Combining formula (1) and Lemma 20 we get the following explicit formula for the price vector $p=p(G)=p(G, b)$ of the associated mechanism $M_{G}$.

Lemma 22 We have $p_{i}=\sum_{T \in \mathcal{T}_{i}} w_{T}(b)$.

The price ratio function $p_{i j}(G)=p_{i}(G) / p_{j}(G)$ can be expressed as a rational function in the variables $b_{k l}$, and by definition (see section 3.3) $\pi_{i j}=$ $\pi_{i j}(G)$ is the number of variables that remain in this expression after all possible cancellations have been taken into account. We will write $\pi(G)=$ $\max _{i j} \pi_{i j}(G)$ for the complexity of the $G$-mechanism.

\subsection{Graphs with complexity $\leq 4$}

If $G$ consists of a single vertex then $\pi(G)=0$ by definition.

Lemma 23 If $G$ is a cycle then $\pi(G)=2$.

Proof. Each vertex $i$ in a cycle has a unique outgoing edge, and we denote its weight by $a_{i}$. For each $i$ we have $p_{i}=b_{G} / a_{i}$; hence $p_{i} / p_{j}=a_{j} / a_{i}$ and the result follows.

By a chorded cycle we mean a graph that is a union $G=C \cup P$ where $C$ is a cycle and $P$, the chord, is a path that connects two distinct vertices of $C$, but which is otherwise disjoint from $C$.

Lemma 24 If $G=C \cup P$ is a chorded cycle then $\pi(G)=4$.

Proof. Let $i$ be the initial vertex of the path $P$, then $i$ has two outgoing edges, $i j$ and $i k$ say, on the cycle and path respectively. Any vertex $l \neq i$ has a unique outgoing edge, and we denote its weight by $a_{l}$ as before. Let $x$ be the terminal vertex of the path $P$. If $x=j$ then $G$ has two $j$-trees, otherwise there is a unique $j$-tree; similarly if $x=k$ then there are two $k$-trees, otherwise there is a unique $k$-tree. Thus we get the following table:

\begin{tabular}{|l|c|c|c|}
\hline & $x=j$ & $x=k$ & $x \neq j, k$ \\
\hline$p_{j} / b_{G}$ & $a_{j}^{-1}\left(b_{i k}^{-1}+b_{i j}^{-1}\right)$ & $a_{j}^{-1} b_{i k}^{-1}$ & $a_{j}^{-1} b_{i k}^{-1}$ \\
\hline$p_{k} / b_{G}$ & $a_{k}^{-1} b_{i j}^{-1}$ & $a_{k}^{-1}\left(b_{i k}^{-1}+b_{i j}^{-1}\right)$ & $a_{k}^{-1} b_{i j}^{-1}$ \\
\hline
\end{tabular}


In every case, the ratio $p_{j} / p_{k}$ depends on all 4 variables $a_{j}, a_{k}, b_{i j}, b_{i k}$, thus $\pi(G) \geq 4$.

On the other hand, since all vertices other than $i$ have a unique outgoing edge, it follows that if $x$ is any vertex then every $x$-tree contains all the outgoing edges except perhaps the edges $b_{i j}, b_{i k}$ and $a_{x}$ (if $x \neq i$ ); thus $p_{x}$ is divisible by all other weights. It follows that for any two vertices $x, y$ the ratio $p_{x} / p_{y}$ can only depend on the variables $b_{i j}, b_{i k}, a_{x}, a_{y}$. Thus we get $\pi(G) \leq 4$ and hence $\pi(G)=4$ as desired.

Remark 25 A special case of a chorded cycle is a graph $T_{0}$ with three vertices that we call a chorded triangle.

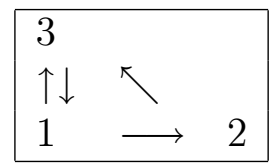

\begin{tabular}{|l|l|}
\hline$p_{1}$ & $b_{23} b_{31}$ \\
\hline$p_{2}$ & $b_{12} b_{31}$ \\
\hline$p_{3}$ & $b_{23}\left(b_{12}+b_{13}\right)$ \\
\hline
\end{tabular}

\begin{tabular}{|l|l|}
\hline$p_{1} / p_{2}$ & $b_{23} / b_{12}$ \\
\hline$p_{2} / p_{3}$ & $b_{12} b_{31} / b_{23}\left(b_{12}+b_{13}\right)$ \\
\hline$p_{3} / p_{1}$ & $\left(b_{12}+b_{13}\right) / b_{31}$ \\
\hline
\end{tabular}

For future use we note that for each index $j$ there is an $i$ such that $\pi_{i j} \geq 3$.

By a $k$-rose we mean a graph that is a union $C_{1} \cup \cdots \cup C_{k}$, where the $C_{i}$ are cycles that share a single vertex $j$, but which are otherwise disjoint. Thus a 0 -rose is a single vertex and a 1 -rose is a cycle. If $G$ is a $k$-rose for some $k \geq 2$ then we will simply say that $G$ is a rose.

If each cycle in a rose $G$ has exactly two vertices, i.e., is a bidirected edge, then we say that $G$ is a star.

Lemma 26 If $G$ is a rose then $\pi(G)=4$.

Proof. Let $G$ be the union of cycles $C_{1} \cup \cdots \cup C_{k}$ with common vertex $j$ as above. Let $a_{1}, \ldots, a_{k}$ be the weights of the outgoing edges from $j$ in cycles $C_{1}, \ldots, C_{k}$ respectively, and for all other vertices $x$ let $b_{x}$ denote the weight of the unique outgoing edge at $x$. It is easy to see that there for each vertex $v$ of $G$ there is a unique $v$-tree, and thus the price vectors are given as follows:

$$
p_{j}=\prod_{x \neq j} b_{x}, \quad p_{x}=\frac{a_{i} p_{j}}{b_{x}} \text { if } x \neq j \text { is a vertex of } C_{i}
$$

Thus we get

$$
p_{j} / p_{x}=b_{x} / a_{i}, \quad p_{y} / p_{x}=b_{x} a_{l} / b_{y} a_{i} \text { if } y \neq j \text { is a vertex of } C_{l}
$$

Taking $i \neq l$, we see that $p_{y} / p_{x}$ depends on 4 variables, and $\pi(G)=4$.

Our main result is a classification of connected graphs with $\pi(G) \leq 4$. 
Theorem 27 If $G$ is not a chorded cycle or a $k$-rose, then $\pi(G) \geq 5$.

We give a brief sketch of the proof of this theorem, which will be carried out in the rest of this section. The actual proof is organized somewhat differently, but the main ideas are as follows.

We say that a graph $H$ is a minor of $G$, if $H$ can be obtained from $G$ by removing some edges and vertices, and collapsing certain kinds of edges. Our first key result is that the property $\pi(G) \leq 4$ is a hereditary property, in the sense that connected minors of such graphs also satisfy the property. The usual procedure for studying a hereditary property is to identify the forbidden minors, namely a set $\Gamma$ of graphs such that $G$ fails to have the property iff it contains one of the graphs from $\Gamma$. We identify a finite collection of such graphs. The final step is to show that if $G$ is not a chorded cycle or a $k$-rose then it contains one of the forbidden minors.

We note the following immediate consequence of the results of this section.

Corollary 28 If $G$ is not a cycle then $\pi_{i j}(G) \geq 4$ for some ij.

\subsection{Subgraphs}

Throughout this section $G$ denotes a connected graph. We say that a graph $H$ is a subgraph of $G$ if $H$ is obtained from $G$ by deleting some edges and vertices.

Proposition 29 If $G^{\prime}$ is a connected subgraph of $G$ then $\pi(G) \geq \pi\left(G^{\prime}\right)$.

Proof. For a vertex $i$ in $G^{\prime}$ let $p_{i}^{\prime}$ and $p_{i}$ denote its price in $G^{\prime}$ and $G$ respectively; we first relate $p_{i}^{\prime}$ to a certain specialization of $p_{i}$.

Let $E, E^{\prime}$ be the edge sets of $G, G^{\prime}$ respectively, and let $E_{0}$ (resp. $E_{1}$ ) denote the edges in $E \backslash E^{\prime}$ whose source vertex is inside (resp. outside) $G^{\prime}$. Let $\bar{p}_{i}$ be the specialization of $p_{i}$ obtained by setting the edge weights in $E_{0}$ and $E_{1}$ to 0 and 1 respectively. Then we claim that

$$
p_{i}^{\prime}=|F| \bar{p}_{i},
$$

where $F$ is the set of directed forests $\phi$ in $G$ such that

1. the root vertices of $\phi$ are contained in $G^{\prime}$,

2. the non-root vertices of $\phi$ consist of all $G$-vertices not in $G^{\prime}$. 
Indeed, consider the expression of $p_{i}$ as a sum of $i$-trees in $G$. The specialization $\bar{p}_{i}$ assigns zero weight to all trees with an edge from $E_{0}$. The remaining $i$-trees in $G$ are precisely of the from $\tau \cup \phi$ where $\tau$ is an $i$-tree in $G^{\prime}$ and $\phi \in F$, and these get assigned weight $w t(\tau)$. Formula (10) is an immediate consequence.

Now if $i, j$ are vertices in $G^{\prime}$, then formula (10) gives

$$
\frac{p_{i}^{\prime}}{p_{j}^{\prime}}=\frac{\bar{p}_{i}}{\bar{p}_{j}}
$$

Thus the $i j$ price ratio in $G^{\prime}$ is obtained by a specialization of the ratio in $G$. Consequently the former cannot involve more variables. Taking the maximum over all $i, j$ we get $\pi(G) \geq \pi\left(G^{\prime}\right)$ as desired.

\subsection{Collapsible edges}

We write $\operatorname{out}(k)$ for the number of outgoing edges at the vertex $k$. In a connected graph we have out $(k) \geq 1$ for all vertices, and we will say $k$ is ordinary if $\operatorname{out}(k)=1$ and special if $\operatorname{out}(k)>1$. Among special vertices, we will say that $k$ is binary if out $(k)=2$ and tertiary if out $(k)=3$.

Definition 30 We say that an edge ij of a graph $G$ is collapsible if

1. $i$ is an ordinary vertex

2. $j i$ is not an edge of $G$

3. there is no vertex $k$ such that $k i$ and $k j$ are both edges of $G$.

Definition 31 If $G$ has no collapsible edges we will say $G$ is rigid.

If $G$ is a connected graph with a collapsible edge $i j$, we define the $i j$ collapse of $G$ to be the graph $G^{\prime}$ obtained by deleting the vertex $i$ and the edge $i j$, and replacing any edges of the form $l i$ with edges $l j$. The assumptions on $i j$ imply that the procedure does not introduce any loops or double edges, hence $G^{\prime}$ is also simple (and connected). Moroever each vertex $k \neq i$ has the same outdegree in $G^{\prime}$ as in $G$.

Lemma 32 If $G^{\prime}$ is the ij-collapse of $G$ as above, then $\pi(G) \geq \pi\left(G^{\prime}\right)$. 
Proof. Let $k$ be any vertex of $G^{\prime}$ then $k$ is also a vertex of $G$. Since $i$ is ordinary every $k$-tree in $G$ must contain the edge $i j$; collapsing this edge gives a $k$-tree in $G^{\prime}$ and moreover every $k$-tree in $G^{\prime}$ arises uniquely in this manner. Thus we have a factorization

$$
p_{k}(G)=a_{i j} p_{k}\left(G^{\prime}\right)
$$

Thus for any two vertices $k, l$ of $G^{\prime}$ we get $p_{k}(G) / p_{l}(G)=p_{k}\left(G^{\prime}\right) / p_{l}\left(G^{\prime}\right)$ and the result follows.

We will say that $H$ is a minor of $G$ if it is obtained from $G$ by a sequence of steps of the following kind: a) passing to a connected subgraph, b) collapsing some collapsible edges. By Proposition 29 and Lemma 32 we get

Corollary 33 If $H$ is a minor of $G$ then $\pi(H) \leq \pi(G)$.

\subsection{Augmentation}

Throughout this section $G$ denotes a connected graph.

Notation 34 We write $H \unlhd G$ if $H$ is a connected subgraph of $G$, and write $H \triangleleft G$ to mean $H \unlhd G$ and $H \neq G$.

We say that $H \triangleleft G$ can be augmented if there is a path $P$ in $G$ whose endpoints are in $H$, but which is otherwise completely disjoint from $H$. We refer to $P$ as an augmenting path of $H$, and to $K=H \cup P$ as an augmented graph of $H$; note that $K$ is also connected, i.e. $K \unlhd G$. It turns out that augmentation is always possible.

Lemma 35 If $H \triangleleft G$ then $H$ can be augmented.

Proof. If $G$ and $H$ have the same vertex set then any edge in $G \backslash H$ comprises an augmenting path. Otherwise consider triples $\left(k, P_{1}, P_{2}\right)$ where $k$ is a vertex not in $H, P_{1}$ is a path from some vertex in $H$ to $k$, and $P_{2}$ is a path from $k$ to some vertex in $H$. Among all such triples choose one with $e\left(P_{1}\right)+e\left(P_{2}\right)$ as small as possible. Then $P_{1}$ and $P_{2}$ cannot share any intermediate vertices with $H$ or with each other, else we could construct a smaller triple. It follows that $P=P_{1} \cup P_{2}$ is an augmenting path.

We are particularly interested in augmenting paths for $H$ that consist of one or two edges; we refer to these as short augmentations of $H$. 
Corollary 36 If $H \triangleleft G$ then $G$ has a minor that is a short augmentation of $H$.

Proof. Let $K=H \cup P$ be an augmentation of $H$. If $P$ has more than two edges, then we may collapse the first edge of $P$ in $K$. The resulting graph is a minor of $G$, which is again an augmentation of $H$. The result follows by iteration.

Lemma 37 If $K=H \cup P$ with $P=\{j k, k l\}$, then for any vertex $i$ of $H$ we have $\pi_{i k}(K)=\pi_{i j}(H)+2$.

Proof. The edges $(j, k)$ and $(k, l)$ are the unique incoming and outgoing edges at $k$. It follows that every $i$-tree in $K$ is obtained by adding the edge $k l$ to an $i$-tree in $H$, and every $k$-tree in $K$ is obtained by adding the edge $j k$ to a $j$-tree in $H$. Thus if $a_{j k}$ and $a_{k l}$ are the respective weights of the two edges in the path $P$ then we have

$$
p_{i}(K)=a_{k l} p_{i}(H), p_{k}(K)=a_{j k} p_{j}(H) \Longrightarrow \frac{p_{i}(K)}{p_{k}(K)}=\frac{a_{k l}}{a_{j k}} \frac{p_{i}(H)}{p_{j}(H)}
$$

Thus the price ratio in question depends on two additional variables, and the result follows.

Corollary 38 If $G$ contains the chorded triangle $T_{0}$ as a proper subgraph then $\pi(G) \geq 5$.

Proof. By the previous corollary $G$ has a minor $K=T_{0} \cup P$, which is a short augmentation of $T_{0}$, and it is enough to show that $\pi(K) \geq 5$. If $P$ consists of two edges $\{j k, k l\}$ then by Remark 25 we can choose $i$ such that $\pi_{i j}\left(T_{0}\right)=3$; now by the previous lemma we have $c_{i k}(K)=5$ and hence $\pi(K) \geq 5$. If $P$ consists of a single edge then $K$ is necessarily as below, and once again $\pi(K) \geq 5$.

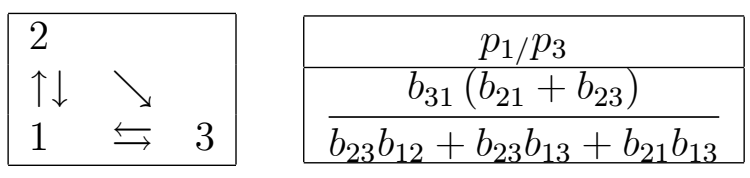




\subsection{The circuit rank}

As usual $G$ denotes a simple connected graph, and we will write $e(G)$ and $v(G)$ for the numbers of edges and vertices of $G$.

Definition 39 The circuit rank of $G$ is defined to be

$$
c(G)=e(G)-v(G)+1
$$

The circuit rank is also known as the cyclomatic number, and it counts the number of independent cycles in $G$, see e.g. [3].

Example 40 If $G$ is a $k$-rose then $c(G)=k$, and if $G$ is a chorded cycle then $c(G)=2$.

We now prove a crucial property of $c(G)$.

Proposition 41 If $H \triangleleft G$ then there is some $K \unlhd G$ such that $H \triangleleft K$ and $c(K)=c(H)+1$.

Proof. Let $K=H \cup P$ be an augmentation of $H$. If $P$ consists of $m$ edges, then $K$ has $e(H)+m$ edges and $v(H)+m-1$ vertices; hence $c(K)=c(H)+1$.

Corollary 42 Let $G$ be a connected graph.

1. If $H \triangleleft G$ then $c(H)<c(G)$.

2. $c(G)=0$ iff $G$ is a single vertex.

3. $c(G)=1$ iff $G$ is a cycle.

4. $c(G)=2$ iff $G$ is a chorded cycle or a 2-rose.

Proof. The first part follows from the previous proposition, the other parts are completely straightforward.

Lemma 43 If $G$ is not a rose and $c(G)>3$, then there is some $K \triangleleft G$ such that $K$ is not a rose and $c(K)=3$. 
Proof. Let $R$ be a $k$-rose in $G$ with $c(R)=k$ as large as possible, then $R \triangleleft G$ by assumption. If $c(R) \leq 2$ then any $K \triangleleft G$ with $c(K)=3$ is not a rose. Thus we may assume that $c(R)>2$, and in particular $R$ has a unique special vertex $i$ and at least three loops. Since $R \neq G, R$ can be augmented, and $S=R \cup P$ is an augmentation, then $P$ cannot both begin and end at $i$, else $R \cup P$ would be a rose, contradicting the maximality of $R$. Since there are at most two endpoints of $P$, we can choose two distinct loops $L_{1}$ and $L_{2}$ of $R$, such that $L_{1} \cup L_{2}$ contains these endpoints of $P$. Then $K=L_{1} \cup L_{2} \cup P$ is the desired graph.

\subsection{Covered vertices}

Definition 44 Let $i$ be an ordinary vertex of $G$ with outgoing edge ij. We say that a vertex $k$ covers $i$, if one of the following holds:

1. the edges $\mathrm{ki}$ and $\mathrm{kj}$ belong to $G$

2. $j=k$ and the edge $k i$ belongs to $G$

If there is no such $k$ then we say that $i$ is an uncovered vertex.

We emphasise that the terminology covered/uncovered is only applicable to ordinary vertices in a graph $G$. The main point of this definition is the following simple observation.

Remark 45 An ordinary vertex is uncovered iff its outgoing edge is collapsible.

Lemma 46 Suppose $G$ is a connected graph.

1. If $v(G) \geq 3$ then an ordinary vertex cannot cover another vertex.

2. If $v(G) \geq 4$ then a binary vertex can cover at most one vertex.

3. A tertiary vertex can cover at most three vertices.

4. If $G$ is a rigid graph with $c(G)=3$, then $v(G) \leq 4$. 
Proof. If $k$ is an ordinary vertex covering $i$ then $G$ must contain the edges $k i$ and $i k$. Thus $i$ and $k$ do not have any other outgoing edges, and if $G$ has a third vertex $j$ then there is no path from $k$ or $i$ to $j$, which contradicts the connectedness of $G$, thereby proving the first statement.

If $k$ is a binary vertex covering the ordinary vertices $i$ and $j$ then $G$ must contain the edges $k i, k j, i j, j i$. The vertices $i, j, k$ cannot have any other outgoing edges, so a fourth vertex would contradict the connectedness of $G$ as before. This proves the second statement.

If a vertex $k$ covers $i$ then there must be an edge from $k$ to $i$. Thus if $\operatorname{out}(k)=3$ then $k$ can cover at most three vertices.

If $c(G)=3$ then $G$ has either 2 binary vertices or 1 tertiary vertex, with the remaining vertices being ordinary. If $v(G)>4$ then by previous two paragraphs $G$ would have an uncovered vertex, which is a contradiction.

\subsection{Proof of Theorem 27}

Proposition 47 If $c(G) \geq 3$ and $G$ is not a rose, then $\pi(G) \geq 5$.

Proof. By Proposition 29 and Lemma 43 we may assume that $c(G)=3$. By Lemma 32, we may further assume that $G$ is rigid, and thus by Lemma 46 that $v(G) \leq 4$. We now divide the argument into three cases.

First suppose that $G$ contains a 3 -cycle $C$. We claim that at least one of the edges of $C$ must be a bidirected edge in $G$, so that $G$ properly contains a chorded triangle $T_{0}$, whence $\pi(G) \geq 5$ by Corollary 38 . Indeed if $G$ has no other vertices outside $C$, then $G$ must have 5 edges and 3 vertices and the claim is obvious. Thus we may suppose that there is an outside vertex $l$. We further claim that $C$ contains two vertices $i, j$ such that $i$ covers $j$. Granted this, it is immediate that $G$ contains either the bidirected edge $i j$ and $j i$, or the bidirected edge $j k$ and $k j$ where $k$ is the third vertex of $C$. To prove the "further" claim we note that the special vertices of $G$ consist of either a) one tertiary vertex, or b) two binary vertices. In case a) the connectedness of $G$ implies that the tertiary vertex must be in $C$, and hence it must cover both the ordinary vertices in $C$. In case b) either $C$ contains both binary vertices, one of which must cover the unique ordinary vertex of $C$; or $C$ contains one binary vertex, which must cover one of the two ordinary vertices of $C$.

Next suppose that $G$ does not contain a 3 -cycle, but does contain a 4cycle labeled 1234, say. Now $G$ has two additional edges, which cannot be the diagonals $13,31,24,42$, since otherwise $G$ would have a 3 -cycle; therefore 
$G$ must have two bidirected edges. The bidirected edges cannot be adjacent else $G$ would have a collapsible vertex, therefore $G$ must be the first graph below, which has $\pi(G) \geq 5$.
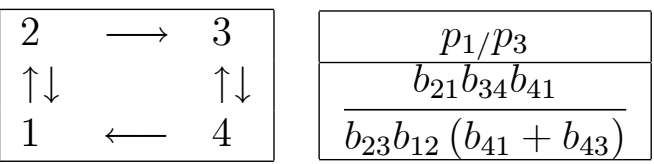

\begin{tabular}{|lll|l|}
\hline 2 & $\rightleftarrows$ & 3 \\
$\uparrow \downarrow$ & & $\uparrow \downarrow$ \\
1 & & 4
\end{tabular}$\quad$\begin{tabular}{c|}
$\frac{p_{1 /} p_{4}}{b_{21} b_{32} b_{43}}$ \\
$\frac{b_{34} b_{23} b_{12}}{2}$
\end{tabular}

Finally suppose $G$ has no 3-cycles or 4-cycles. Then every edge must be a bidirected edge, and $G$ must be a tree with all bidirected edges. Since $G$ is not a star, this only leaves the second graph above, which has $\pi(G) \geq 6$.

We can now finish the proof of Theorem 27.

Proof of Theorem 27. If $c(G) \leq 2$ then by the previous corollary, $G$ is a single vertex, a cycle, chorded cycle or a 2-rose. If $c(G) \geq 3$ then the result follows by the previous proposition.

\section{Proof of Theorem 12}

In this section, after a couple of preliminary results, we apply Theorem 27 to prove Theorem 12 .

Lemma 48 If $G$ is a chorded cycle on 4 or more vertices, then $\tau(G) \geq 3$.

Proof. We can express $G$ as a union of two paths $P, Q$ from 1 to 2 , say and a third path $R$ from 2 to 1 . At least one of the first two paths, say $P$ must have an intermediate vertex, say 3 . Since $m \geq 4$ there is an additional intermediate vertex 4 on one of the paths.

If $m=4$ then we get three possible graphs depending on the location of the vertex 4 .

$$
\begin{array}{lll}
3 & \rightarrow & 4 \\
\uparrow & & \downarrow \\
1 & \leftrightarrows & 2
\end{array} \quad \quad \begin{array}{lll}
3 & \rightarrow & 2 \\
\uparrow & \nearrow & \downarrow \\
1 & \leftarrow & 4
\end{array} \quad \quad \begin{array}{llll}
3 & \rightarrow & 2 \\
\uparrow & \swarrow & \uparrow \\
1 & \rightarrow & 4 \\
\hline
\end{array}
$$

For these graphs we have $\tau_{24}=3, \tau_{42}=3$ and $\tau_{34}=3$, respectively. Thus $\tau(G) \geq 3$ in all three cases.

If $m>4$ then $G$ can be realized as one of these graphs, albeit with additional intermediate vertices on one or more of the paths $P, Q, R$. These additional vertices are ordinary uncovered vertices, with collapsible outgoing 
edges. Collapsing one of these edges does not increase time complexity, and produces a smaller chorded cycle $G^{\prime}$. Arguing by induction on $m$ we conclude $\tau(G) \geq \tau\left(G^{\prime}\right) \geq 3$

Lemma 49 If $G$ is the complete graph, then $\pi_{i j}(G)=m(m-1)$ for all $i \neq j$.

Proof. Fix a pair of vertices $i \neq j$ in $G$. Then we claim that the price ratio $p_{i j}(G)$ depends on each of the $m(m-1)$ edge weights $b_{k l}$. Indeed if $H$ is any "spanning" connected subgraph of $G$ then $p_{i j}(H)$ is obtained from $p_{i j}(G)$ by specializing to 0 the weights of all edges outside $H$. Therefore it suffices to find a connected subgraph $H$ such that $p_{i j}(G)$ depends on $b_{k l}$.

We consider two cases. If $\{i, j\}=\{k, l\}$ then exchanging $i, j$ if necessary we may assume $i=k, j=l$. Let $H$ be an $m$-cycle two of whose edges are $i j$ and $h i$ (say); then $p_{i} / p_{j}=b_{h i} / b_{i j}$ depends on $b_{k l}=b_{i j}$.

If $\{i, j\} \neq\{k, l\}$ then let $H$ be an 2-rose with loops $C_{1}$ and $C_{2}$ such that

1. $k$ is the special vertex, and $k l$ is an edge in $C_{1}$

2. $i$ belongs to $C_{1}$ and $j$ belongs to $C_{2}$

Then $p_{i}$ and $p_{j}$ are each given by unique directed trees $T_{i}$ and $T_{j}$. Moreover $T_{i}$ involves $k l$ while $T_{j}$ does not. Hence $p_{i j}(H)$ depends on $b_{k l}$.

Before proceeding further it will be helpful to recall some basic ordertheoretic notions.

Definition 50 A quasiorder $\precsim$ on a set $X$ is a binary relation that is reflexive $(x \precsim x)$ and transitive:

$$
x \precsim y, y \precsim z \Longrightarrow x \precsim z
$$

We write $x \prec y$ if $x \precsim y$ holds but $y \precsim x$ does not hold. We say that $x$ is $\precsim$-minimal if there is no $y$ in $X$ such $y \prec x$, equivalently if for all $y \in X$

$$
y \precsim x \Longrightarrow x \precsim y \text {. }
$$

We write $X_{\prec}$ for the set of $\precsim$-minimal elements of $X$. We say that $\precsim$ is a well-quasiorder (wqo) if there does not exist an infinite descending chain

$$
\cdots \prec x_{n} \prec \cdots \prec x_{2} \prec x_{1} \text {. }
$$


Note that if $\precsim$ is a wqo on $X$ and $Y \subset X$ then the restriction of $\precsim$ defines a wqo on $Y$. In general the minimal elements $Y_{\prec}$ can be quite different from $X_{\prec}$, however we have the following elementary result.

Lemma 51 If $(X, \precsim)$ is a wqo and $X_{\prec} \subset Y \subset X$ then $X_{\prec}=Y_{\prec}$.

Proof. Any minimal element of $X$ that happens to lie in $Y$ is clearly minimal in $Y$. Thus $X_{*} \subset Y$ implies $X_{\prec} \subset Y_{\prec}$. On the other hand if $z$ is a non-minimal element of $X$ then $x \prec z$ for some $x \in X_{\prec}$, otherwise we could construct an infinite descending chain in $X$ starting with $z$. In particular any $z \in Y \backslash X_{\prec}$ satisfies $x \prec z$ for some $x \in X_{\prec} \subset Y$, hence $z$ is not minimal in $Y$.

It is easy to check that both the quasiorders $\preceq$ and $\preceq_{w}$ that we have introduced on $\mathfrak{M}$ are wqo's; and therefore in the proof below we will apply the previous lemma to them.

Proof of Theorem 12. Let $\mathfrak{S}$ denote the set consisting of the three special mechanisms. We need to show that $\left(\mathfrak{M}_{g}\right)_{\prec_{w}}=\mathfrak{S}$ and $\left(\mathfrak{M}_{*}\right)_{\prec_{w}}=\mathfrak{S}$.

We first prove that $\left(\mathfrak{M}_{g}\right)_{\prec_{w}}=\mathfrak{S}$. Let us say that $G$ is a strongly minimal graph if $M_{G}$ is a strongly minimal mechanism of $\mathfrak{M}_{g}$. Now the star mechanism has complexity $(\tau, \pi)=(2,4)$. Therefore if $G$ is any strongly minimal graph then either $\tau(G)=1$ or $\pi(G) \leq 4$. For $\tau(G)=1$ we get the complete graph, which has complexity $(\tau, \pi)=(1, m(m-1))$ by Lemma 49 . The graphs with $\pi(G) \leq 4$ are characterized by Theorem 27 , and we have three possibilities for $G$.

1. Chorded cycle. In this case we have $(\tau, \pi)=\left(3^{+}, 4\right)$ by Lemma 48 , and so $G$ is not strongly minimal.

2. Cycle. In this case we have $(\tau, \pi)=(m-1,2)$ by Lemma 23 .

3. $k$-rose, $k \geq 2$. If each petal of $G$ has exactly 2 edge then $G$ is the star mechanism. Otherwise after collapsing edges, we obtain the minor

$$
\begin{array}{lllll}
\hline 1 & & & & \\
\downarrow & \nwarrow & & & \\
\cdot & \rightarrow & . & \leftrightarrows & 2
\end{array} \quad \text { with } \tau_{12}=3
$$

Thus $G$ has complexity $(\tau, \pi)=\left(3^{+}, 4\right)$ and so is not strongly minimal. 
Thus the three graphs in the statement of Theorem 12 are the only possible strongly minimal graphs, and have the indicated complexities. Since they are incomparable with each other, each is strongly minimal. Thus we conclude $\left(\mathfrak{M}_{g}\right)_{\prec w}=\mathfrak{S}$ as desired.

We now prove $\left(\mathfrak{M}_{*}\right)_{\prec_{w}}=\mathfrak{S}$. Since $\left(\mathfrak{M}_{g}\right)_{\prec_{w}}=\mathfrak{S}$, by Lemma 51 applied to the wqo $\preceq_{w}$ it suffices to show that $\mathfrak{S} \subset \mathfrak{M}_{*}$. We further note that

$$
\mathfrak{M}_{*}=\left(\mathfrak{M}_{g}\right)_{\prec} .
$$

Indeed $\mathfrak{M}_{*}=\mathfrak{M}_{\prec}$ by definition, and $\mathfrak{M}_{*} \subset \mathfrak{M}_{g}$ by Theorem 11; now (11) follows from Lemma 51 applied to the wqo $\preceq$. Thus it suffices to prove that

$$
\mathfrak{S} \subset\left(\mathfrak{M}_{g}\right)_{\prec}
$$

i.e., that each of the three special mechanisms is $\preceq$-minimal in $\mathfrak{M}_{g}$.

The $\preceq$-minimality is obvious for the complete graph since any other graph would have some $\tau_{i j}>1$, and also for the cycle since any other graph would have some $k_{i}>1$. To establish $\preceq$-minimality for the star graph, it suffices to show that any non-star graph $G$ has either some $\pi_{i j} \geq 5$ or some $\tau_{i j} \geq 3$. For this we note that $\pi \geq 5$ holds by Theorem 27 if $G$ is not a rose or a chorded cycle; while $\tau \geq 3$ holds trivially for non-star roses and by Lemma 48 for chorded cycles. This completes the proof of $(12)$ and hence of $\left(\mathfrak{M}_{*}\right)_{\prec w}=\mathfrak{S}$.

Remark 52 For $m=3$, Lemma 48 does not hold and we have an additional strongly minimal mechanism with $(\tau, \pi)=(2,4)$, namely the chorded triangle

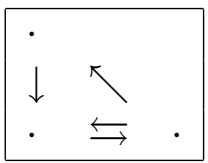

\section{A Continuum of Traders}

Our analysis easily extends to the case where the set of individuals $T$ is the unit interval $[0,1]$, endowed with a nonatomic population measure ${ }^{18}$. Let $\mathcal{S}$ denote the collection of all integrable functions $\boldsymbol{a}: T \mapsto S$ such that $\int_{T} \boldsymbol{a} \in$

\footnotetext{
${ }^{18}$ Denote the measure $\mu$. And since $\mu$ is to be held fixed throughout, we may suppress it, abbreviating $\int_{T} \mathbf{f}(t) d \mu(t)$ by $\int_{T} \mathbf{f}$ for any measurable function $\mathbf{f}$ on $[0,1]$.
} 
$S_{+}$. (An element of $\mathcal{S}$ represents a choice of offers by the traders in $T$ which are positive on aggregate.) In the same vein, let $\mathcal{R}$ denote the collection of all integrable functions from $T$ to $C$, whose elements $\mathbf{r}: T \mapsto C$ represent returns to $T$. An exchange mechanism $M$, on a given set of $m$ commodities, is a map from $\mathcal{S}$ to $\mathcal{R}$ such that, if $M$ maps $\boldsymbol{a}$ to $\mathbf{r}$ then we have (reflecting conservation of commodities):

$$
\int_{T} \boldsymbol{a}=\int_{T} \mathbf{r}
$$

We wrap the aggregation and anonymity conditions into one, and directly postulate that the return to any individual depends only on his own offer and the integral of everyone's offers, and that this return function is the same for everyone. Thus we have a function $r$ from $S \times S_{+}$to $C$ such that $\mathbf{r}(t)=r(a, b)$, where $a=\mathbf{a}(t)$ and $b=\int_{T} \boldsymbol{a}$.

The rest of the analysis is exactly the same (with obvious modifications in the notation, occasioned by the continuum). The only difference is in the proof of the fact that $r$ is linear in the first factor and homogeneous of degree 0 in the second, which proceeds as follows.

Proposition 53 (Linearity). For any fixed $b, r(a, b)$ is a linear function of a.

Proof. (This simple argument is as in [Dubey-MasColell-Shubik].) We will first show that if $a, c \in S$ and $0<\lambda<1$, then

$$
r(\lambda a+(1-\lambda) c, b)=\lambda r(a, b)+(1-\lambda) r(c, b)
$$

There clearly exists an integrable map $\mathbf{d}$ from $T=[0,1]$ to space of offers $S$ such that (i) positive mass of traders choose $a$ in $\mathbf{d}$; (ii) positive mass of traders choose $c$ in $\mathbf{d}$; and (iii) the integral of $\mathbf{d}$ on $T$ is $b$. So $\int_{T} r\left(\mathbf{d}^{\alpha}, b\right) d \mu(\alpha)=\int_{T} r(\mathbf{d}, b)=\bar{b}$ since commodities are conserved. Shift $\varepsilon \lambda$ mass from $a$ to $\lambda a+(1-\lambda) c$ and $(1-\lambda) \varepsilon$ mass from $c$ to $\lambda a+(1-\lambda) c$, letting the rest be according to $\mathbf{d}$. This yields a new function (from $T$ to $S$ ) which we call e. Clearly the integral of e on $T$ is also $b$. Therefore, once again by conservation of commodities, we must have $\int_{T} r(\mathbf{e}, b)=\bar{b}$, hence $\int_{T} r(\mathbf{d}, b)=$ $\int_{T} r(\mathbf{e}, b)$. But this can only be true if the displayed equality holds, proving that (every coordinate of) $r$ is affine in $a$ for fixed $b$.

Now $r(0, b) \geq 0$ by assumption. Suppose $r(0, b) \gtreqless 0$. Partition $T$ into two non-null sets $T_{1}$ and $T_{2}$. Consider the case where all the individuals in $T_{1}$ 
offer 0 , and all in $T_{2}$ offer $b / \mu\left(T_{2}\right)$. Then, since everone in $T_{1}$ gets the return $r(0, b) \gtreqless 0$, by conservation of commodities everyone in $T_{2}$ gets $\bar{b}-\mu\left(T_{1}\right)$ $r(0, b) \lesseqgtr b / \mu\left(T_{2}\right)$, contradicting non-dissipation. So $r(0, b)=0$, showing $r$ is linear.

Proposition 54 (Homogeneity) $r(a, \lambda b)=r(a, b)$ for any $a, b$ and positive scalar $\lambda$

Proof. This follows from $\lambda r(a, b)=r(\lambda a, \lambda b)=\lambda r(a, \lambda b)$, where the first equality comes from invariance and the second from the Linearity Proposition.

Remark 55 As mentioned in the introduction, when there is a continuum of traders, the star mechanism leads to equivalence (or, near-equivalence) of Nash and Walras equilibria under suitable postulates regarding the commodity or fiat money. (See [7] for a detailed discussion.)

\section{References}

[1] A. Abel and B. Bernanke (2005). Macroeconomics, Pearson, p 266-269.

[2] Bannerjee, A.V. and E. Maskin (1996). A Walrasian theory of money and barter, Quarterly Journal of Economics, 111(4): 955-1005.

[3] Berge, Claude (2001), Cyclomatic number, The Theory of Graphs, Courier Dover Publications, pp. 27-30.

[4] Dubey, P. and M. Shubik (1978). The noncooperative equilibria of a closed trading economy with market supply and bidding strategies. Journal of Economic Theory, 17 (1): 1-20.

[5] Dubey, P., A. Mas-Colell and M. Shubik (1980). Efficiency properties of strategic market games: an axiomatic approach. Journal of Economic Theory, 22 (2): 363-76

[6] Dubey, P. (1982) Price-quantity strategic market games Econometrica, 50 (1): 111-126.

[7] Dubey, P. and L. S. Shapley (1994). Noncooperative general exchange with a continuum of traders. Journal of Mathematical Economics, 23: 253-293. 
[8] Dubey, P. and S. Sahi (2003). Price-mediated trade with quantity signals. Journal of Mathematical Economics, Special issue on strategic market games (in honor of Martin Shubik), ed. G. Giraud, 39: 377-389

[9] Dubey, P. and J. Geanakoplos (2003). From Nash to Walras via ShapleyShubik. Journal of Mathematical Economics, Special issue on strategic market games (in honor of Martin Shubik), ed. G. Giraud, 39: 391-400

[10] Dubey, P. and J. Geanakoplos (2003). Inside and outside fiat money, gains to trade, and IS-LM. Economic Theory, 21(2-3): 347-497.

[11] Foley, D.K. (1970). Economic equilibrium with costly marketing. Journal of Economic Theory, 2(3): 276-91.

[12] Hahn, F. H. (1971). Equilibrium with transactions costs. Econometrica, 39 (3): 417-39.

[13] Heller, W. P. (1974). The holding of money balances in general equilibrium. Journal of Economic Theory, 7: 93-108.

[14] Heller, W.P. and R. Starr. Equilibrium with non-convex transactions costs: monetary and non-monetary economies. Review of Economic Studies, 43 (2): 195-215.

[15] Howitt, P. and R. Clower. (2000). The emergence of economic organization. Journal of Economic Behavior and Organization, 41: 55-84

[16] Iwai, K. (1996). The bootstrap theory of money: a search theoretic foundation for monetary economics. Structural Change and Economic Dynamics, 7: 451-77

[17] Jevons, W.S. (1875). Money and the mechanism of exchange. London: D. Appleton

[18] Jones, R.A.(1976). The origin and development of media of exchange: Journal of Political Economy, 84: 757-75

[19] Knapp, G.F. (1905) Staatliche Theorie des Geldes, 4th edition, Munich and Leipzig: Duncker \& Humblot. Translated as The State Theory of Money, London: Macmillan, 1924. 
[20] Kiyotaki, N and R. Wright (1989). On money as a medium of exchange. Journal of Political Economy, 97: 927-54

[21] Kiyotaki, N and R. Wright (1993). A search-theoretic approach to monetary economics. American Economic Review, 83 (1): 63-77

[22] Krugman,P. and R. Wells (2006). Economics, Worth Publishers, New York.

[23] Kaulla, R. (1920) Grundlagen des geldwerts, Stuttgart. Translation in Howard S. Ellis, German Monetary Theory: 1903-1933, Cambridge, MA: Harvard University Press, 1934.

[24] Lerner, A. P. (1947). Money as a creature of the state. In Proceedings of the American Economic Association, Vol 37: 312-17

[25] Li, Y. and R. Wright (1998). Government transaction policy, media of exchange, and prices. Journal of Economic Theory, 81 (2): 290-313

[26] Mertens, J. F. (2003). The limit-price mechanism. Journal of Mathematical Economics, Special issue on strategic market games (in honor of Martin Shubik), ed. G. Giraud, 39: 433-528.

[27] Menger, C. (1892). On the origin of money. Economic Journal, 2: 23955, trans. Caroline A. Foley

[28] Milnes, A. (1919). The economic foundations of reconstruction, Macdonald and Evans, p 55.

[29] Ostroy, J.M. (1973). The informational efficiency of monetary exchange, American Economic Review,63 (4): 597-610.

[30] Ostroy, J. and R. Starr. (1974). Money and the decentralization of exchange. Econometrica, 42: 597-610

[31] Ostroy, J. and R. Starr. (1990). The transactions role of money. In B. Friedman and F. Hahn (eds) Handbook of Monetary Economics, New York: Elsevier, North- Holland: 3-62.

[32] Peck, J., K. Shell and S. E. Spear (1992). The market game: existence and structure of equilibria. Journal of Mathematical Economics 
[33] Peck, J. and K. Shell (1992). Market uncertainty: correlated sunspot equilibria in imperfectly competitive economies. The Review of Economic Studies, 1992

[34] Postlewaite, A. and D. Schmeidler (1978). Approximate efficiency of non-Walrasian equilibria. Econometrica, 46 (1): 127-36.

[35] Sahi, S. (2013). Harmonic vectors and matrix tree theorems. arXiv preprint arXiv:1309.4047.

[36] Sahi, S. and S. Yao. (1989). The noncooperative equilibria of a closed trading economy with complete markets and consistent prices. Journal of Mathematical Economics, 18: 325-346.

[37] Schumpeter, J. A. (1954). History of Economic Analysis. New York: Oxford University Press.

[38] Shapley, L. S. (1976). Noncooperative general excchange. In Theory and Measurement of Economic Externalities. Academc Press (ed) A. Y. Lin: $155-175$.

[39] Shapley, L. S. and M. Shubik (1977). Trade using one commodity as a means of payment. Journal of Political Economy, 85: 937-968.

[40] Shubik, M. (1973). Commodity money, oligopoly, credit and bankruptcy in a general equilibrium model. Western Economic Journal, 11: 24-38.

[41] Shubik, M. and C. Wilson (1977). The optimal bankruptcy rule in a trading economy using fiat money. Zeitschrift fur Nationalokonomie, 37 (3-4): 337-354.

[42] Shubik, M. (1999). The theory of money and financial institutions. Cambridge, MA: MIT Press.

[43] Starr, R.M. (2012). Why is there money? Edward Elgar, Cheltenham, UK \& Northhampton, MA, US.

[44] Starret, D.A. (1973). Inefficiency and the demand for money in a sequence economy. Review of Economic Studies,40 (4): 437-48

[45] Trejos, A. and R.Wright (1995). Search, bargaining, money and prices. Journal of Political Economy, 103(1) : 118-41. 
[46] Wallace, N. (1980). The overlapping generations model of fiat money. In J. Karaken and N. Wallace, Models of Monetary Economics, Minneapolis, MN: Federal Reserve Bank of Minneapolis: 49-82 